\title{
Study of the Transfer Between Libration Point Orbits and Lunar Orbits in Earth-Moon System
}

\author{
Yu Cheng ${ }^{1,2}$. Gerard Gómez ${ }^{2}$. \\ Josep J. Masdemont ${ }^{3}$. Jianping Yuan ${ }^{1}$
}

Received: date / Accepted: date

\begin{abstract}
This paper is devoted to the study of the transfer problem from a libration point orbit of the Earth-Moon system to an orbit around the Moon. The transfer procedure analysed has two legs: the first one is an orbit of the unstable manifold of the libration orbit and the second one is a transfer orbit between a certain point on the manifold and the final lunar orbit. There are only two manoeuvres involved in the method and they are applied at the beginning and at the end of the second leg. Although the numerical results given in this paper correspond to transfers between halo orbits around the $L_{1}$ point (of several amplitudes) and lunar polar orbits with altitudes varying between 100 and 500 $\mathrm{km}$, the procedure we develop can be applied to any kind of lunar orbits, libration orbits around the $L_{1}$ or $L_{2}$ points of the Earth-Moon system, or to other similar cases with different values of the mass ratio.
\end{abstract}

Keywords Transfer orbits · libration point orbits · invariant manifolds · lunar orbits

\section{Introduction}

Since some years ago, missions from different space agencies have revisited the Moon with several kinds of spacecraft and devices including lunar orbiters, landers, rovers, or sample return spacecraft. A Lunar $L_{1}$ or $L_{2}$ Gateway Station could

\footnotetext{
Yu Cheng · Jianping Yuan

1 National Key Laboratory of Aerospace Flight Dynamics, Northwestern Polytechnical University, Xian 710072, PR China

E-mail: cheng@maia.ub.es

Gerard Gómez

2 IEEC \& Department de Matemàtiques i Informàtica, Universitat de Barcelona, Gran Via 585, 08007 Barcelona, Spain

Josep J. Masdemont

${ }^{3}$ IEEC \& Department de Matemàtiques, Universitat Politècnica de Catalunya, Diagonal 647, 08028 Barcelona,Spain
} 
support infrastructures beyond orbits around the Earth and serve as a staging location for missions to the Moon (see [14]).

Several approaches have been used for the analysis of these missions, depending on the different goals to be achieved (see, for instance, [3, $\mathbf{5}, \mathbf{8}, \mathbf{1 6}, 17,19]$ ). These include the acquisition of accurate and high-resolution 3D maps of the surface for the selection of future landing sites, the exploration of possible water resources near its poles, testing new technologies, etc. Some of these lunar missions, such as Artemis or Chang'e 2, have used libration point orbits (LPO) as their nominal target trajectory or they have visited this kind of orbits in the Earth-Moon and the Earth-Sun systems.

As it is well known, in the circular restricted three-body problem (CR3BP), or in any perturbation of it, libration point orbits around the $L_{1}$ and $L_{2}$ points can be easily reached from the vicinity of the small primary. This fact is because the stable manifolds of these libration point orbits have close approaches to the small primary or even intersect it. Main examples of applicability consider the Earth departure in the Sun-Earth system, or the Moon in the Earth-Moon system.

Furthermore, since there exist heteroclinic connections between the libration point orbit of the Sun-Earth and Earth-Moon systems, it is possible to transfer from one system to the other [12,13, or in other scenarios, to obtain low energy transfer paths to visit other Solar System bodies. This kind of connections were used by the Chinese Chang'e 2 spacecraft 6, 15. Chang'e 2 was launched in October 2010 to conduct research from a $100 \mathrm{~km}$ high lunar polar orbit and provided high-resolution images of the lunar surface. In June 2011 Chang'e 2 left the lunar orbit towards the Sun-Earth $L_{2}$ Lagrangian point that was reached in August 2011, after a 77-day cruise. Although it was expected to remain at $L_{2}$ until the end of 2012, in April 2012 Chang'e 2 departed on an extended mission to flyby the asteroid 4179 Toutatis at a distance of $3.2 \mathrm{~km}$ in December of the same year [6].

Considering the framework of the circular restricted three-body problem (CR3BP), in this paper we analyse the transfer from a libration point orbit (LPO) around a collinear equilibrium point to an orbit around the small primary. The analysis of these trajectories, which in some sense are the inverse of the transfers usually considered in the literature [18, enhance the possibilities to be considered for missions relating libration point orbits.

The procedure is developed and explained in a general context, but most of the computations are done for the transfer case between halo orbits around the $L_{1}$ point of the Earth-Moon system, with different $z$-amplitudes, and polar orbits around the Moon (taking into account the orientation of the rotation axis of the Moon) with altitudes varying between 100 and $500 \mathrm{~km}$. Nevertheless, it can also be applied to compute transfers from any kind of libration point orbit, around $L_{1}$ or $L_{2}$, of an arbitrary CR3BP model, to circular Keplerian orbits around the small body of the system.

As general conclusions we can say that, in order to optimize the transfer strategy, it is convenient to depart at a point along the unstable manifold of the LPO at a distance from the Moon between 45, $000 \mathrm{~km}$ and $20,000 \mathrm{~km}$ and to perform the change of inclination, to reach one of the desired lunar orbit, at this point. The size of the departing halo orbit has little influence in the total cost, and of course the cost depends and decreases when increasing the altitude of the target lunar orbit. In 
order to shorten the time of flight, the departure manoeuvre should be done at the first apolune of the unstable invariant manifold of the LPO.

Even that in this paper we do not address the cost of the transfer in a high realistic force field model (including, for instance, the gravitational effect of the Sun or the eccentricity of the Orbit of the Moon) or the influence of the departing epoch from the LPO. We have checked that modulus of the relative residual accelerations between the CR3BP and a $n$-body model (defined by the JPL ephemerids) is of the order of 0.0012, independently of the transfer orbit and the initial epoch. So the qualitative and quantitative results for real transfers should be close to the ones given in this work, with estimated variations in the total cost of less than $10 \%$.

The paper is organized as follows: in Section 2 the reference model, changes of coordinates and the notation used in the paper are introduced. In Section 3 we explain the transfer procedure and how the computation of the two transfer manoeuvres we use is done. The numerical results for the transfer between halo orbits of the Earth-Moon system and lunar polar orbits are given in Section 4. Finally, the last Section contains a summary of conclusions.

\section{The dynamic model}

Along this paper, we use the circular restricted three body problem as dynamic model for the motion of a spacecraft in the Earth-Moon system, this is: with the mass ratio $\mu$ equal to 0.012150582 (according to the DE401 ephemeris data file). In the synodic reference frame, the origin is set at the barycentre of the system, the positive $x$-axis is pointing from the Moon to the Earth, the $z$-axis is in the direction of the normal vector to the Moon's orbit around the Earth, and the $y$-axis completes the right-hand coordinate system. In the usual non-dimensional system, in which the length-unit is the semi-axis of the Moon's orbit around the Earth and the time-unit is such that the period of the Moon around the Earth is $2 \pi$, one unit of non-dimensional distance is of the order of $0.3844037 \times 10^{6} \mathrm{~km}$ and one time unit is approximately $0.377496 \times 10^{6} \mathrm{sec}$.

According to 22 , the differential equations of the model can be written as

$$
\begin{aligned}
\ddot{x}-2 \dot{y} & =\Omega_{x}, \\
\ddot{y}+2 \dot{x} & =\Omega_{y}, \\
\ddot{z} & =\Omega_{z},
\end{aligned}
$$

where $\Omega(x, y, z)=\left(x^{2}+y^{2}\right) / 2+(1-\mu) / r_{1}+\mu / r_{2}+\mu(1-\mu) / 2, r_{1}=\sqrt{(x-\mu)^{2}+y^{2}+z^{2}}$ and $r_{2}=\sqrt{(x-\mu+1)^{2}+y^{2}+z^{2}}$. The CR3BP has a first integral given by $\mathcal{C}=2 \Omega(x, y)-\dot{x}^{2}-\dot{y}^{2}-\dot{z}^{2}$, where $\mathcal{C}$ is the so called Jacobi's integral.

It is well known that the CR3BP differential equations (1) have three collinear equilibrium (or libration) points, $L_{1}, L_{2}$ and $L_{3}$, and the two equilateral ones, $L_{4}$ and $L_{5}$. If $x_{L_{i}}$ denotes the $x$ coordinate of the $L_{i}$ point, then $x_{L_{2}} \leq \mu-1 \leq x_{L_{1}} \leq$ $\mu \leq x_{L_{3}}$.

According to the values of the eigenvalues associated to the collinear equilibrium points, $\pm i \lambda_{1}, \pm i \lambda_{2}, \pm \lambda_{3}$, with $\lambda_{1,2,3} \in \mathbb{R}$ (see [22]), the three points are centre $\times$ centre $\times$ saddle critical points. Due to the centre $\times$ centre part (associated to 
the imaginary eigenvalues $\pm i \lambda_{1}$ and $\pm i \lambda_{2}$ ), and considering all the energy levels, there are $4 \mathrm{D}$ centre manifolds around these points that, for a given value of the Jacobi constant, are just 3D sets. The saddle component of the flow (associated to the real eigenvalues $\pm \lambda_{3}$ ) makes the dynamics around the libration points that of an unstable equilibrium. For values of the Jacobi constant close to that of $L_{1}$ and $L_{2}$, the unstable and stable manifolds associated to the periodic orbits and invariant tori rule the dynamics in the neighbourhood of the equilibrium points (see 11,14]).

2.1 Change of coordinates between the synodic CR3BP and the Moon-centred sidereal frames

In this section we briefly describe the change of coordinates that has been used to transform the usual synodic coordinates of the CR3BP and the Keplerian orbital elements, $(a, i, e, \Omega, \omega, f)$ in a Moon-centred sidereal frame $\left\{M-x^{\prime} y^{\prime} z^{\prime}\right\}$, where $f$ stands for the true anomaly.

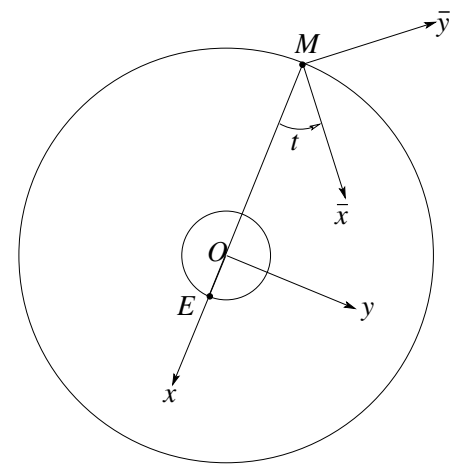

Fig. 1: Relation between the usual CR3BP synodic reference frame $\{O-x y z\}$, and the intermediate Moon-centred sidereal reference frame $\{M-\bar{x} \bar{y} \bar{z}\}$. The $z$ axis of both systems is perpendicular to the plane displayed.

Let $\left(x^{s y n}, y^{s y n}, z^{s y n}\right)$ be the synodic coordinates of a point $\boldsymbol{r}^{\text {syn }}$ in the CR3BP synodic reference frame, $\{O-x y z\}$, with origin $O$ at the Earth-Moon barycentre (see Figure 1). A translation $\boldsymbol{d}$ along the $x$-axis of modulus $1-\mu$ sets the origin at the Moon, and a rotation around the $z$ axis of angle $n t, R_{3}(t)$, transforms the synodic coordinates to an intermediate sidereal reference frame $\{M-\bar{x} \bar{y} \bar{z}\}$, which is aligned with $\{O-x y z\}$ at an initial epoch $t_{0}$. In this way, the transformation between the synodic and the sidereal system is given by:

$$
\boldsymbol{r}^{0}=R_{3}(t)\left(\boldsymbol{r}^{\text {syn }}+\boldsymbol{d}\right)=\left(\begin{array}{ccc}
\cos (t) & -\sin (t) & 0 \\
\sin (t) & \cos (t) & 0 \\
0 & 0 & 1
\end{array}\right)\left(\begin{array}{c}
1-\mu \\
0 \\
0
\end{array}\right) .
$$

The inclination $i$ of the orbit of a spacecraft around the Moon is measured with respect to the Moon's equator. For this purpose, we introduce the Mean 
Earth/Polar Axis Lunar reference system $\left\{M-x^{\prime} y^{\prime} z^{\prime}\right\}$, which, according to [1] (see Figure 2), is defined as follows: the origin is located at the centre of the Moon, the $z^{\prime}$-axis is aligned with the mean Moon's rotation axis (orthogonal to the Moon's equator) at a certain epoch $t_{0}$, and the Prime Meridian ( $0^{\circ}$ lunar longitude) is the mean Earth direction at the same epoch, so the $x^{\prime}$-axis points towards the mean Earth at $t_{0}$, the $y^{\prime}$-axis completes the right-hand coordinate system. Due to the inclination of the Moon's equator with respect to the Moon's orbital plane around the Earth $((\bar{x}, \bar{y})$ plane $)$, the $z^{\prime}$ axis forms an angle of approximately $6.7^{\circ}$ with the $\bar{z}$-axis. Due to the long precession period of the Moon's rotation axis (of about 18.6 years) we assume that $z^{\prime}$-axis is fixed during a mission lifetime.

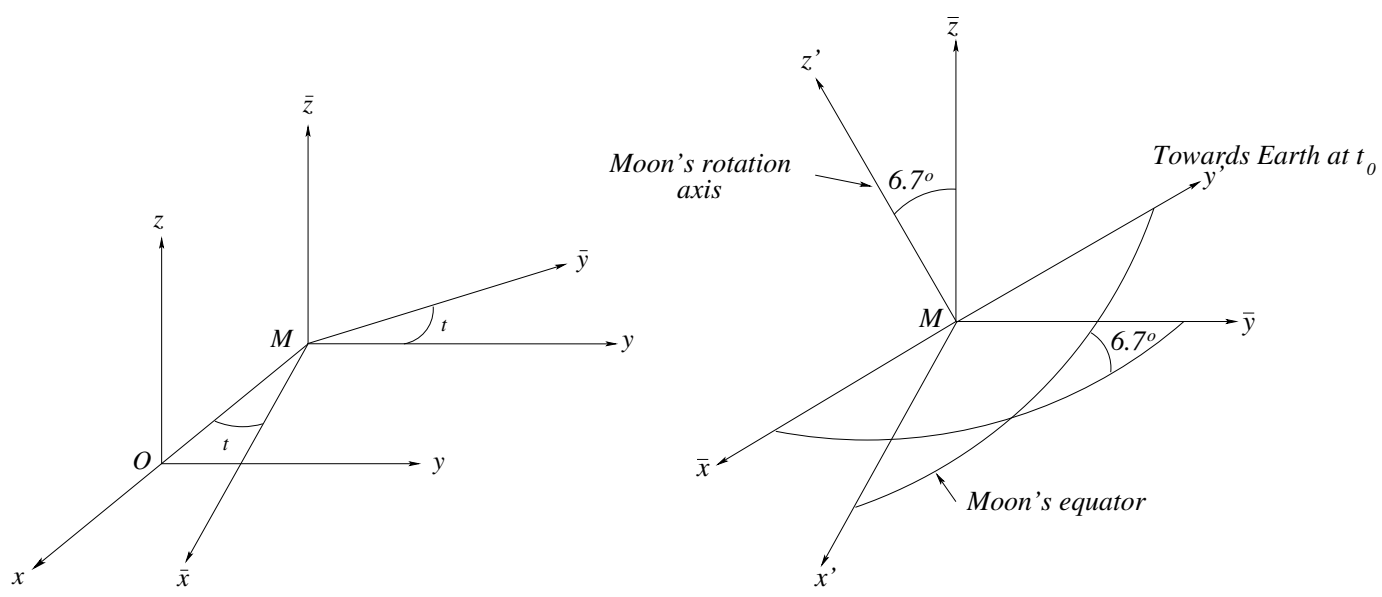

Fig. 2: Moon's centred sidereal reference frames. The left plot displays the relation between the $\{M-\bar{x} \bar{y} \bar{z}\}$ reference system and the usual CR3BP synodic reference frame $\{O-x y z\}$. Both frames are parallel at $t_{0}$. The right hand side plot displays the two inertial Moon centred reference frames: $\{M-\bar{x} \bar{y} \bar{z}\}$ and $\left\{M-x^{\prime} y^{\prime} z^{\prime}\right\}$. The inclination $i$ of an orbit around the Moon is measured with respect to the $\left(x^{\prime}, y^{\prime}\right)$ plane.

To compute the change of coordinates between the intermediate sidereal reference frame $\{M-\bar{x} \bar{y} \bar{z}\}$ and the sidereal reference frame $\left\{M-x^{\prime} y^{\prime} z^{\prime}\right\}$, we use an additional frame, ICRF, which is the one used in the JPL ephemeris file DE421. If $\boldsymbol{r}^{\text {sid }}$ and $\boldsymbol{r}^{i c r f}$ denote the coordinates of a point in the reference frames $\left\{M-x^{\prime} y^{\prime} z^{\prime}\right\}$ and $I C R F$, respectively, the relation between both is

$$
\boldsymbol{r}^{\text {sid }}=B \boldsymbol{r}^{i c r f}
$$

where the matrix $B$ depends on the the lunar libration angles at the initial epoch $t_{0}$ (see 4] for details).

If $\boldsymbol{r}^{0}$ denotes the coordinates of the point $\boldsymbol{r}^{i c r f}$ in the $\{M-\bar{x} \bar{y} \bar{z}$ frame, then, according to [4], we have

$$
\boldsymbol{r}^{i c r f}=C \boldsymbol{r}^{0}
$$


where the columns of the matrix $C$ are: $\boldsymbol{r}_{m},\left(\boldsymbol{r}_{m} \times \boldsymbol{v}_{m}\right) \times \boldsymbol{v}_{m}$, and $\boldsymbol{r}_{m} \times \boldsymbol{v}_{m}$. The vectors $\boldsymbol{r}_{m}$ and $\boldsymbol{v}_{m}$ are the position and velocity of the Moon relative to the Earth at the epoch $t_{0}$, respectively (and can be also obtained from DE421).

In this way, we get the transformation between the two inertial reference frames $\left\{M-x^{\prime} y^{\prime} z^{\prime}\right\},\{M-\bar{x} \bar{y} \bar{z}\}$ and the synodic reference frame $\{O-x y z\}$ is:

$$
\boldsymbol{r}^{\text {sid }}=B C \boldsymbol{r}^{0}=B C R_{3}(t)\left(\boldsymbol{r}^{\text {sid }}+\boldsymbol{d}\right) .
$$

The matrix $A=B C R_{3}(t)$ depends on the initial epoch. Using the data of the ephemeris file DE421, and as initial reference date $t_{0}$ the epoch 2020 Jan. 1 12:00:00(TDB)

$$
A=\left(\begin{array}{rrr}
0.99338553 & -0.00636032 & -0.11465040 \\
0.00250881 & 0.99942862 & -0.03370651 \\
0.11479928 & 0.03319592 & 0.99283390
\end{array}\right)
$$

These are the values that have been used for the numerical results given in Section 4. Although we have not done a systematic study of the results as a function of, some explorations show that the variations in the $\Delta v$ costs are small (usually less than $5 \%$ ).

To simplify the notation, from now on we will avoid the use of the super-index sid and syn when the context is clear enough.

\section{Computation of a transfer from libration point orbit to a circular lunar orbit}

In this section we explain the general procedure used to compute transfer trajectories from a libration point orbit around the $L_{1}$ point of the Earth-Moon system (such as a halo orbit) to a circular lunar orbit with a certain inclination $i$. For the explanation of the method we assume that the LPO and the orbit around the Moon (determined by its radius) are fixed.

In the procedure, the transfer trajectory has two legs. The first one, the manifold leg, goes from $P_{0}$ to $P_{1}$ and follows an orbit of the unstable manifold of the libration orbit (in fact, of the branch of the manifold that starts going towards the Moon). The second one, the Hohmann leg, goes from $P_{1}$ to $P_{2}$, and is a Hohmann like transfer orbit that, after a suitable manoeuvre $\Delta \boldsymbol{v}_{1}$ at $P_{1}$ connects a point of the unstable manifold with the lunar orbit at $P_{2}$, where it does an insertion manoeuvre $\Delta \boldsymbol{v}_{2} . P_{1}$ and $P_{2}$ are the apocentre and pericentre of the Hohmann leg, respectively. The whole transfer process is sketched in Figure 3

Assuming that no $\Delta v$ is required to depart from the libration orbit, since the inherent instability of the orbit does this task for us, the full process requires two manoeuvres $\Delta \boldsymbol{v}_{1}$ and $\Delta \boldsymbol{v}_{2}$. The first one is required to depart from the orbit of the unstable manifold, and we call it the departure manoeuvre. The second manoeuvre $\Delta \boldsymbol{v}_{2}$, namely the insertion manoeuvre, inserts the spacecraft into an orbit around the Moon. In principle, the final goal is to minimise the total transfer cost in terms of $\Delta v_{t o t}=\left\|\Delta \boldsymbol{v}_{1}\right\|+\left\|\Delta \boldsymbol{v}_{2}\right\|$, in this paper we explore the role of the free parameters of the problem in the value of $\Delta v_{t o t}$.

We assume that we move along an orbit of the unstable manifold of the departure orbit during $\Delta t_{1}$ time units, reaching the state $P_{1}=\left(\boldsymbol{r}_{m}^{s y n}, \boldsymbol{v}_{m}^{s y n}\right)$ in the 


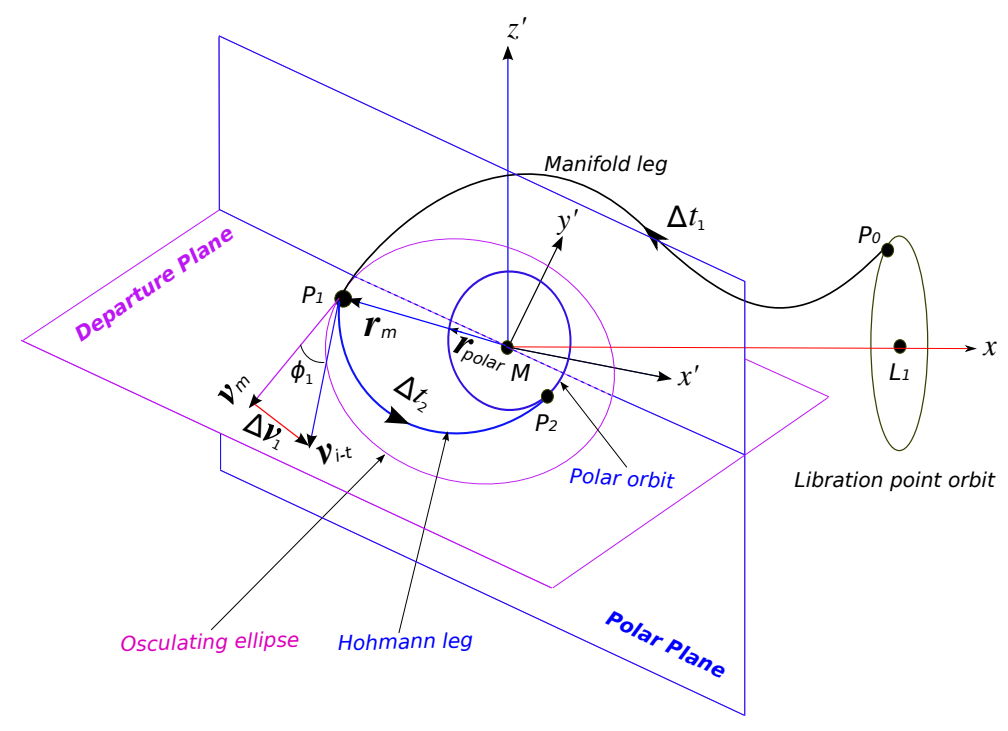

Fig. 3: Sketch of whole transfer process. The transfer starts at the point $P_{0}$ of a libration point orbit. From $P_{0}$ to $P_{1}$ (manifold leg) it follows an orbit of the unstable manifold of the libration orbit. The first transfer manoeuvre $\Delta v_{1}$ is performed at $P_{1}$ at a distance $r_{m}$ from the Moon. At this point the spacecraft is injected in the Hohmann leg contained in the plane of the final target orbit. The second transfer manoeuvre, not represented in the figure, is done at $P_{2}$, where the spacecraft is injected in a lunar polar orbit of radius $r_{\text {polar }}$.

synodic system $\{O-x y z\}$. At this point, after transforming it to the sidereal system $\left\{M-x^{\prime} y^{\prime} z^{\prime}\right\}$, we perform the first manoeuvre $\Delta \boldsymbol{v}_{1}$ to reach the polar lunar orbit by means of a non-coplanar Hohmann transfer.

To compute $\Delta \boldsymbol{v}_{1}$, we need to look at $P_{1}$ in a two body problem (2BP) framework around the Moon. Let $\left(\boldsymbol{r}_{m}, \boldsymbol{v}_{m}\right)$ be the coordinates of this point in sidereal reference frame $\left\{M-x^{\prime} y^{\prime} z^{\prime}\right\}$, computed using the transformation between the sidereal and the synodic systems, together with its derivative. Note that, the position vector $\boldsymbol{r}_{m}$ is aligned with the intersection line between departure plane and polar plane.

The point $\left(\boldsymbol{r}_{m}, \boldsymbol{v}_{m}\right)$ defines a Keplerian osculating orbit around the Moon (represented by the purple dashed line in Figure 3 contained in the departure plane) whose associated Keplerian elements are $\left(a_{m}, i_{m}, e_{m}, \Omega_{m}, w_{m}, f_{m}\right)$. In general, the inclination $i_{m}$ is not the one of the target lunar orbit. The computation of the suitable $\Delta \boldsymbol{v}_{1}$ which performs the transfer to a lunar orbit with a given inclination $i$ is explained later.

After the first manoeuvre, $\Delta \boldsymbol{v}_{1}$, the state vector becomes $\left(\boldsymbol{r}_{i-t}=\boldsymbol{r}_{m}, \boldsymbol{v}_{i-t}=\right.$ $\boldsymbol{v}_{m}+\Delta \boldsymbol{v}_{1}$ ), which is the initial state of the transfer orbit (represented by blue dashed line in Figure 3 contained in the polar plane). This point is propagated during $\Delta t_{2}$ time units until it reaches a sphere centred at the Moon with radius $r_{\text {polar }}$. In the $\{O-x y z\}$ reference system the sphere is defined as $S=$ 
$\left\{(x, y, z) \mid(x+1-\mu)^{2}+y^{2}+z^{2}-r_{\text {polar }}^{2}=0\right\}$. At this point the second manoeuvre $\Delta \boldsymbol{v}_{2}$ is performed in order to get captured into a circular orbit around the Moon with the desired inclination. All the propagations are done in the synodic reference frame using the CR3BP equations (1).

In conclusion, the problem is essentially a non-coplanar Hohmann transfer where the location of the places of the two manoeuvres are not specified in advance. In a first approximation we assume that these places are the apocentre and pericentre of a non-coplanar Hohmann-like transfer ellipse, from a certain point of the unstable manifold to a non-coplanar circular orbit around the Moon of radius $r_{\text {polar. }}$. As it has already been said, the main problem is to determine where and how to perform the two manoeuvres in order to minimise $\Delta v_{t o t}=\left\|\Delta \boldsymbol{v}_{1}\right\|+\left\|\Delta \boldsymbol{v}_{2}\right\|$.

3.1 Computation of manoeuvres to transfer to a Keplerian ellipse with a fixed inclination $i$

As it has been stated, the first manoeuvre deals with the change of inclination between the osculating ellipse associated with a certain point $P_{1}$ of the unstable manifold and the circular lunar orbit. In this section we explain the general method to compute the manoeuvre that injects the spacecraft into a Keplerian ellipse contained in a plane with a fixed inclination $i$. The method is similar to the one developed in [7] and [20].

In general, to guarantee that a spacecraft with initial conditions $(\boldsymbol{r}, \boldsymbol{v})$ moves on a given target plane defined by its normal vector $\boldsymbol{n}$, its initial velocity $\boldsymbol{v}$ must be contained in the plane, this is $\boldsymbol{v} \perp \boldsymbol{n}$. Next we are going to explain how to compute the modulus of $\boldsymbol{v}$ and its direction.

The inclination $i$ of the target plane is the angle between the normal to this plane $\boldsymbol{n}$ and the $z^{\prime}$-axis. Therefore, $\boldsymbol{n}$ is on a cone of angle $2 i$ around $z^{\prime}$ axis with vertex at the Moon (see Figure 4). Moreover, $\boldsymbol{n}$ is also in a plane perpendicular to $\boldsymbol{r}$. As a consequence, it is the intersection of both surfaces, which in general, are two lines defined by the unitary vectors $\boldsymbol{n}^{1}$ and $\boldsymbol{n}^{2}$.

Let $\boldsymbol{n}=\left(n_{x}, n_{y}, n_{z}\right)$ be a unitary vector perpendicular to $\boldsymbol{r}=(x, y, z)$, so $\boldsymbol{r} \cdot \boldsymbol{n}=0$. We require to the velocity $\boldsymbol{v}_{K}$ of the Keplerian orbit departing from $\boldsymbol{r}$ to be in the plane defined by $\boldsymbol{n}$. The modulus of $\boldsymbol{v}_{K}$ can be determined by the vis-viva equation for a Keplerian ellipse with semi-major axis $a$,

$$
v_{K}=\sqrt{2 \frac{G M}{r}-\frac{G M}{a}},
$$

where $G$ is the gravitational constant, $M$ is the mass of the Moon and $r=\|\boldsymbol{r}\|$.

If $\boldsymbol{r}$ is the apo/pericentre of the ellipse, $\boldsymbol{v}_{K}$ must be perpendicular to $\boldsymbol{n}$ and $\boldsymbol{r}$, so

$$
\boldsymbol{v}_{K}=v_{K} \frac{\boldsymbol{n} \times \boldsymbol{r}}{\|\boldsymbol{n} \times \boldsymbol{r}\|} .
$$

In summary, the conditions that must be fulfilled by a unitary vector $\boldsymbol{n}$ orthogonal to the plane containing $\boldsymbol{r}$ and $\boldsymbol{v}_{K}$ are:

$$
\boldsymbol{r}^{T} \cdot \boldsymbol{n}=0, \quad \boldsymbol{n} \cdot z^{\prime}=\cos i, \quad \boldsymbol{n}^{T} \cdot \boldsymbol{n}=1,
$$

from which we get:

$$
n_{x}=\frac{-b \pm \sqrt{b^{2}-4 a c}}{2 a}, \quad n_{y}=-\frac{n_{x} x+z \cos i}{y}, \quad n_{z}=\cos i
$$




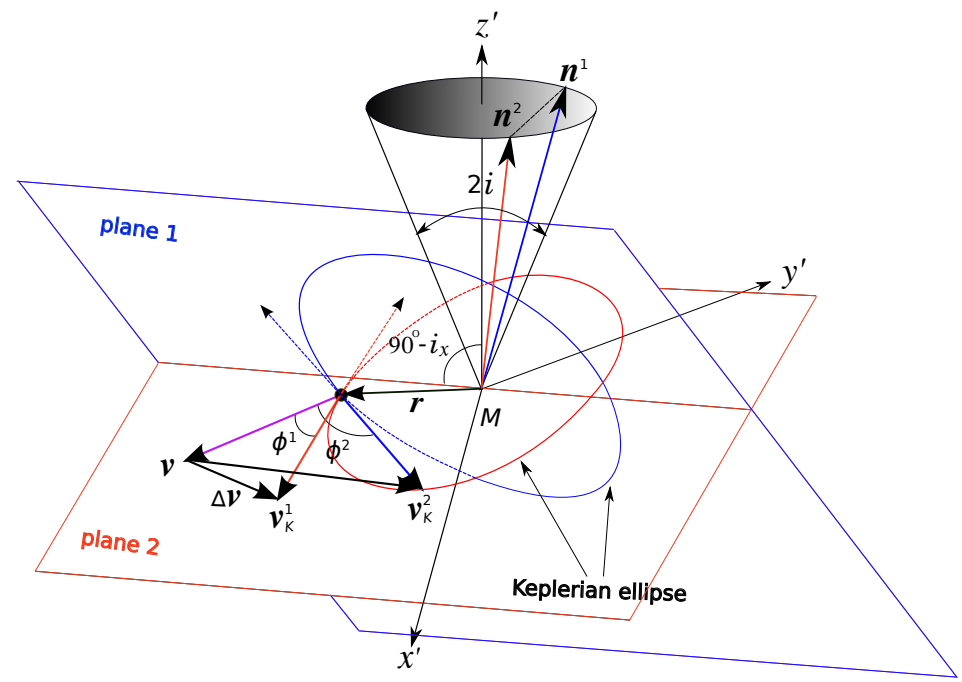

Fig. 4: Intersection of the cone of amplitude $2 i$ around the $z^{\prime}$ axis and the plane perpendicular to $\boldsymbol{r}$. The intersecting directions $\boldsymbol{n}^{1}$ and $\boldsymbol{n}^{2}$ define the two planes shown in the figure. We display here the case when $i \in\left(i_{x}, 90^{\circ}\right)$ for which we have two possible $\boldsymbol{v}_{K}^{1,2}$. For $i \in\left(90,180^{\circ}-i_{x}\right)$ the velocities are the two dashed arrows aligned with $\boldsymbol{v}_{K}^{1,2}$. Note that when $i=i_{x}$ (or $i=180^{\circ}-i_{x}$ ), $\boldsymbol{n}^{1}$ and $\boldsymbol{n}^{2}$, as well as the two velocities $\boldsymbol{v}_{K}^{1,2}$, coincide, and when $i=90^{\circ}$, then $\boldsymbol{v}_{K}^{1,2}$ are aligned but pointing in opposite directions.

where, $a=x^{2}+y^{2}, b=2 x z \cos i, c=z^{2} \cos ^{2} i-y^{2} \sin ^{2} i$. Clearly, we must require that $b^{2}-4 a c \geq 0$, from which it follows that:

$$
\sin ^{2} i \geq \frac{z^{2}}{x^{2}+y^{2}+z^{2}}=\sin ^{2} i_{x} \quad \Rightarrow \quad \cos (2 i) \leq \cos \left(2 i_{x}\right) \quad \Rightarrow \quad i_{x} \leq i \leq 180^{\circ}-i_{x}
$$

where $i_{x}$ in the above inequality (5) is the complementary of the angle between the position vector $\boldsymbol{r}$ and the $z^{\prime}$-axis, which is also the angle between $\boldsymbol{r}$ and the $\left(x^{\prime}, y^{\prime}\right)$ plane.

When the inclinations $i$ and $i_{x}$ do not fulfil the above inequalities, there are no intersections between the cone and the plane perpendicular to $\boldsymbol{r}$ (see Figure 4 for $\left.i \in\left[i_{x}, 90^{\circ}\right]\right)$. Therefore, the available range of values for $i$ is $\left[i_{x}, 180^{\circ}-i_{x}\right]$.

In general, there are two solutions for $\boldsymbol{n}$ and, according to Equation 4 , we have two different $\boldsymbol{v}_{K}$, in the plane defined by $\boldsymbol{n}^{1}$ (plane 1 in Figure 4 ) and by $\boldsymbol{n}^{2}$ (plane 2 in Figure 44, respectively. The required manoeuvres for the transfer are given by:

$$
\Delta v=v_{K}-v
$$

We note that, if $i=i_{x}$ (or $180^{\circ}-i_{x}$ ), the two velocities $\boldsymbol{v}_{K}^{1}$ and $\boldsymbol{v}_{K}^{2}$ coincide and thus we only have one possible $\Delta \boldsymbol{v}$. Also when $i=90^{\circ}, \boldsymbol{v}_{K}^{1}$ and $\boldsymbol{v}_{K}^{2}$ are aligned but pointing in opposite directions. 
3.2 Computation of the departure manoeuvre: first approximation

Next we are going to apply the method described in the previous section to the determination of a first approximation of the first manoeuvre $\Delta \boldsymbol{v}_{1}$.

The first manoeuvre is performed at the apocentre of the (Hohmann) transfer ellipse, so the modulus of the velocity after the manoeuvre must be

$$
v_{i-t}=\sqrt{2 \frac{G M}{r_{m}}-2 \frac{G M}{r_{\text {polar }}+r_{m}}},
$$

where $G$ is the gravitational constant, $M$ is the mass of the Moon. We have used that the pericentre of the ellipse is on the polar orbit around the Moon with radius $r_{\text {polar }}$, so the semi-major axis of this ellipse is $a_{m}=\left(r_{\text {polar }}+r_{m}\right) / 2$.

Since we want to reach a polar orbit around the Moon, the final inclination must be equal to $90^{\circ}$, but for the first manoeuvre, we explore all the suitable values $i_{1} \in\left[i_{x}, 180^{\circ}-i_{x}\right]$ of the inclination of the transfer ellipse. We have seen that for each value of $i_{1}$, there are two possible values of $\boldsymbol{n}$ and, as a consequence, two feasible velocities $\boldsymbol{v}_{i-t}^{1,2}$, both of them must be considered for the determination of the first manoeuvre $\Delta \boldsymbol{v}_{1}=\boldsymbol{v}_{i-t}^{1,2}-\boldsymbol{v}_{m}$. Note that the computation of $\Delta \boldsymbol{v}_{1}$ is done in the sidereal system $\left\{M-x^{\prime}, y^{\prime}, z^{\prime}\right\}$, and also that its modulus is the same in both the sidereal and the synodic system. This is because the transformation between both systems is orthogonal. The two values $\boldsymbol{v}_{i-t}^{1,2}$, that will be refined later, are used as the initial seed for the computation of the departure manoeuvre $\Delta \boldsymbol{v}_{2}$.

Denote $\phi_{1}$ as the angle between the two sidereal velocities at $P_{1}, \boldsymbol{v}_{m}$ and $\boldsymbol{v}_{i-t}$ (displayed in Figure 3). In a first approximation, the modulus of the departure manoeuvre $\Delta v_{1}$ can be written as:

$$
\Delta v_{1}=\sqrt{v_{m}^{2}+v_{i-t}^{2}-2 v_{m} v_{i-t} \cos \phi_{1}},
$$

where $v_{m}=\sqrt{2 \frac{G M}{r_{m}}-\frac{G M}{a_{m}}}$, and $P_{1}$ is considered a point in the osculating ellipse with semi-major axis as $a_{m}$, as defined before. From Equation 8 it follows that the main factors to be considered for the determination of $v_{m}, v_{i-t}$ and $\phi_{1}$ are:

1. The point $P_{1}$

- The distance from $P_{1}$ to the centre of the Moon $r_{m}$. According to equation 7), for a fixed polar radius, $v_{i-t}$ depends only on this distance.

- The inclination of the osculating ellipse at $P_{1}$, which in fact affects the change of inclination $\Delta i_{1}=\left|i_{1}-i_{m}\right|$, and thus $\phi_{1}$.

- The velocity $\boldsymbol{v}_{m}$ of the spacecraft at $P_{1}$.

2. The inclination of the transfer ellipse $i_{1}$, which essentially affects $\phi_{1}$.

3. The departing libration point orbit, since $P_{1}$ is in the unstable manifold associated to it.

We recall that the modulus of $\boldsymbol{v}_{i-t}$ is specified by the vis-viva Equation 7 , but its direction varies with $i_{1}$ and, subsequently, the angle $\phi_{1}$ varies. Of course, $\phi_{1}$ depends also on the inclination of the osculating ellipse at $P_{1}$. It must be noted that $\phi_{1}$, although it is related to the angle between the departure and the polar planes, is not exactly equal to this angle. This is due to the fact that the manifold leg is not a $2 \mathrm{BP}$ orbit, so the osculating inclination along it varies and, depending on the point $P_{1}$ the difference between $\phi_{1}$ and $\Delta i_{1}$ can be large. 
3.3 Refinement of the departure manoeuvre and determination of $P_{2}$

As mentioned before, the insertion manoeuvre at the point $P_{2}$ is done at a point on the sphere $S$ previously defined, and it is also at the pericentre of the transfer ellipse (in a two-body problem scenario). We denote by $\boldsymbol{r}_{f-t}$ and $\boldsymbol{v}_{f-t}$ the sidereal position and velocity of the Hohmann ellipse at its pericentre. By means of the inverse of the transformation between the sidereal and synodic systems, together with its derivative, we obtain the corresponding position and velocity in the synodic system: $\boldsymbol{r}_{f-t}^{s y n}$ and $\boldsymbol{v}_{f-t}^{s y n}$.

To fulfil the above two conditions, the following equations must be satisfied:

$$
\boldsymbol{v}_{f-t}^{s y n} \cdot \nabla S=0, \quad \boldsymbol{r}_{f-t}^{s y n} \cdot \boldsymbol{v}_{f-t}^{s y n}=0
$$

where $S(x, y, z)=(x+1-\mu)^{2}+y^{2}+z^{2}-r_{\text {polar }}^{2}$. We rewrite the second equation as $g=\boldsymbol{r}_{f-t}^{s y n} \cdot \boldsymbol{v}_{f-t}^{\text {syn }}$.

The refinement of the first manoeuvre is iterative, it has two steps and provides also the point $P_{2}$ where the second manoeuvre is done. The first step is to integrate the initial seed given by the Hohmann transfer once it has been converted into the synodic system, $P_{1}=\left(\boldsymbol{r}_{i-t}^{s y n}, \boldsymbol{v}_{i-t}^{s y n}\right)$, until the condition $g=\boldsymbol{r}^{\text {syn }} \cdot \boldsymbol{v}^{\text {syn }}=0$ is fulfilled. This gives us $\Delta t_{2}$, the approximate time of flight along the transfer orbit and a final point $P_{2}=\left(\boldsymbol{r}_{f-t}^{s y n}, \boldsymbol{v}_{f-t}^{s y n}\right)$. To have $g=0$ (with an accuracy of $10^{-12}$ ) we integrate until we get the first change of sign of $g$, and then we apply Newton's method with respect to the transfer time $\Delta t_{2}$.

Since we are integrating the initial condition $\left(\boldsymbol{r}_{i-t}^{s y n}, \boldsymbol{v}_{i-t}^{s y n}\right)$ using the CR3BP equations, in general, when we arrive at $P_{2}$ after $\Delta t_{2}$ time units

$$
S\left(\boldsymbol{r}_{f-t}^{s y n}\right) \neq 0,
$$

because $\boldsymbol{r}_{f-t}^{s y n}$ has been computed in a two-body problem approximation. Note that, in fact, $\boldsymbol{r}_{f-t}^{\text {syn }}=\boldsymbol{r}_{f-t}^{\text {syn }}\left(\boldsymbol{r}_{i-t}^{s i d}, \boldsymbol{v}_{i-t}^{\text {sid }}\right)$, so

$$
S\left(\boldsymbol{r}_{f-t}^{\text {syn }}\right)=\hat{S}\left(\boldsymbol{r}_{f-t}^{\text {syn }}\left(\boldsymbol{r}_{i-t}^{\text {sid }}, \boldsymbol{v}_{i-t}^{\text {sid }}\right)\right)=\hat{S}\left(\boldsymbol{r}_{i-t}^{\text {sid }}, \boldsymbol{v}_{i-t}^{\text {sid }}\right),
$$

and the above condition can be also written as

$$
\hat{S}\left(\boldsymbol{r}_{i-t}^{\text {sid }}, \boldsymbol{v}_{i-t}^{\text {sid }}\right) \neq 0 \text {. }
$$

Now we want to modify the initial condition at $P_{1}$ keeping the position $\boldsymbol{r}_{i-t}^{\text {sid }}$ fixed as well as the direction of $\boldsymbol{v}_{i-t}^{\text {sid }}$ in order that the transfer ellipse remains in a transfer plane with the desired inclination. Then, if we remove those arguments of $\hat{S}$ that are kept fixed, in fact we need to solve

$$
\hat{S}\left(\boldsymbol{v}_{i-t}^{\text {sid }}(1+\Delta v)\right)=0
$$

where now the only free parameter is $\Delta v$.

The value of $\Delta v$ can be obtained using Newton's method, from which we get

$$
\Delta v=-\frac{\hat{S}\left(\boldsymbol{v}_{i-t}^{s i d}\right)}{D \hat{S}_{v_{x}} v_{x}+D \hat{S}_{v_{y}} v_{y}+D \hat{S}_{v_{z}} v_{z}},
$$


where $\boldsymbol{v}_{i-t}^{\text {syn }}=\left(v_{x}, v_{y}, v_{z}\right)$ and $D \hat{S}_{v_{x}}, D \hat{S}_{v_{y}}$ and $D \hat{S}_{v_{z}}$ are components of the matrix $D \hat{S}$ (see [2]). Using $\boldsymbol{X}_{i}^{s y n}=\left(\boldsymbol{r}_{i-t}^{s y n}, \boldsymbol{v}_{i-t}^{s y n}\right), \boldsymbol{X}_{f}^{s y n}=\left(\boldsymbol{r}_{f-t}^{s y n}, \boldsymbol{v}_{f-t}^{\text {syn }}\right)$ one can write

$$
D \hat{S}=\left[\frac{\partial S}{\partial \boldsymbol{r}_{f}^{s y n}} \cdot(\Phi+F \cdot D t) \cdot \frac{\partial \boldsymbol{X}_{i}^{s y n}}{\partial \boldsymbol{X}_{i}^{s i d}}\right],
$$

where $\Phi=\frac{\partial \boldsymbol{X}_{f}^{s y n}}{\partial \boldsymbol{X}_{i}^{s y n}}$ is given by the state transition matrix of CR3BP, $F=\frac{\partial \boldsymbol{X}_{f}^{s y n}}{\partial t_{f}}$ is the CR3BP vector-field, $D t=\frac{\partial \Delta t_{2}}{\partial \boldsymbol{X}_{i}^{s y n}}=-\frac{D g \cdot \Phi}{D g \cdot F}$, and

$$
\frac{\partial \boldsymbol{X}_{i}^{s y n}}{\partial \boldsymbol{X}_{i}^{s i d}}=\left(\begin{array}{c|c}
A & 0 \\
A \cdot J \mid A
\end{array}\right)
$$

where the matrix $A$ is given by $[2]$, and $J=\dot{R}_{3}\left(\Delta t_{1}\right)$.

Once the value of $\Delta v$ has been computed, after one iteration of Newton's method, we go to the first step. The iterative procedure finishes when the value of $\hat{S}$ is less than a certain tolerance (that we have fixed equal to $10^{-12}$ ).

\subsection{Computation of the insertion manoeuvre at $P_{2}$}

The aim of the insertion manoeuvre $\Delta \boldsymbol{v}_{2}$ is to move from the transfer ellipse previously determined to a circular polar orbit of radius $r_{\text {polar }}$ around the Moon. It must be noted that at $P_{2}$ the vector $\boldsymbol{r}_{f-t}^{s y n} \times \boldsymbol{v}_{f-t}^{s y n}$ is not, in general, orthogonal to the polar plane, so $\Delta \boldsymbol{v}_{2}$ accounts for this (small) correction of inclination together with the insertion into a polar orbit. The general procedure to do these manoeuvres is the one that has been explained in Section 3.1. We follow it to compute $\Delta v_{2}$, recalling that we obtain two solutions for $\boldsymbol{v}_{c}^{\text {sid }}$ with the same modulus and opposite directions. Then, we choose the one for which $\Delta v_{2}$ is minimum. It must also be noted that the location of $P_{2}$ can be constrained by mission requirements or by a suitable value of the argument of the ascending node $\Omega$ of the orbit; in this paper almost all the possibilities have been explored in order that the most suitable selection, according to the constraints, can be done.

The modulus of the velocity in a circular polar orbit is given by:

$$
v_{c}^{\text {sid }}=\sqrt{\frac{G M}{r_{\text {polar }}}}
$$

and its inclination with respect to the Moon's equator is $i_{2}=90^{\circ}$.

The value of $\Delta v_{2}$ is obtained from

$$
\Delta \boldsymbol{v}_{2}=\boldsymbol{v}_{c}^{s i d}-\boldsymbol{v}_{f-t}^{s i d} .
$$

and, as in the previous case, the procedure can be summarised as:

$$
\boldsymbol{v}_{f-t}^{\text {syn }} \stackrel{T}{\longrightarrow} \boldsymbol{v}_{f-t}^{\text {sid }} \stackrel{\Delta \boldsymbol{v}_{2}}{\longrightarrow} \boldsymbol{v}_{c}^{\text {sid }}
$$

Similar to what has been said about the factors that influence the value of the manoeuvre $\Delta v_{1}$ at $P_{1}$, we denote by $\phi_{2}$ the angle between the two sidereal 
velocities at $P_{2}, \boldsymbol{v}_{f-t}$ and $\boldsymbol{v}_{c}$. Then, the modulus of the insertion manoeuvre $\Delta v_{2}$ can be written as:

$$
\Delta v_{2}=\sqrt{v_{c}^{2}+v_{f-t}^{2}-2 v_{c} v_{f-t} \cos \phi_{2}},
$$

where, as explained in Section 3.1, $P_{2}$ is the pericentre of the transfer ellipse, which gives us an approximate value $v_{f-t}=\sqrt{2 \frac{G M}{r_{\text {polar }}}-\frac{G M}{r_{\text {polar }}+r_{m}}}$. Although it is refined later by the procedure, it provides a rough estimate of $\Delta v_{2}$ that mainly depends on $r_{\text {polar }}, r_{m}$ and $\phi_{2}$.

If $\boldsymbol{r}_{f-t}^{\text {sid }}=(x, y, z)$ denotes the coordinates of the position of $P_{2}$, in the $\{M-$ $\left.x^{\prime} y^{\prime} z^{\prime}\right\}$ reference frame, then its longitude $\beta$ and latitude $\lambda$ of $\boldsymbol{r}_{f-t}^{\text {sid }}$, with respect to the Moon, are given by

$$
\beta=\arctan \frac{y}{x+1-\mu}, \quad \lambda=\arctan \frac{z}{\sqrt{(x+1-\mu)^{2}+y^{2}}} .
$$

These two values will be used in the description of the numerical results.

\section{Numerical results}

This section is devoted to explain the numerical results we obtain for the transfer methodology explained in the previous section. We have done a systematic exploration of the transfer problem varying those parameters that affect the total velocity cost $\Delta v_{t o t}=\Delta v_{1}+\Delta v_{2}$. These parameters are:

- The $z$-amplitude of the departing halo orbit.

- The point $P_{1}$ at which the departure manoeuvre is performed.

- The inclination $i_{1}$ to be achieved by $\Delta \boldsymbol{v}_{1}$ at $P_{1}$.

- The altitude of the circular polar orbit.

We remark that another parameter that could be considered is the change of inclination $\Delta i_{2}$ to be achieved by $\Delta \boldsymbol{v}_{2}$ at $P_{2}$, which is also the angle between the incoming and outgoing velocities at $P_{2}$; nevertheless the value of $\Delta i_{2}$ is not independent of the above parameters since it is mainly determined by $i_{1}$.

\subsection{Departing halo orbits and their invariant manifolds}

We have used as departing trajectories halo orbits of class I around the libration point $L_{1}$, whose sense of revolution about $L_{1}$ is clockwise as seen from the Moon (see 21]).

The computation of these orbits has been done by means the Lindstedt-Poincaré (LP) procedure (see $[9,10$ ), and the initial conditions obtained have been refined using Newton's method. A sample of the orbits obtained is shown in Figure 5, and the corresponding initial conditions are given in Table 1 .

The computation of the stable and unstable manifolds of the halo orbits has been done using their linear approximation given by eigenvalues and eigenvectors of the monodromy matrix. If $\boldsymbol{x}_{h}$ is the initial condition of the halo orbit and $\boldsymbol{w}^{u}$ is a unitary eigenvector along the unstable direction at this point, then

$$
\boldsymbol{x}_{0}^{u}=\boldsymbol{x}_{h} \pm \epsilon \boldsymbol{w}^{u}
$$


Table 1: Initial conditions $(x, 0, z, 0, \dot{y}, 0)$ and periods $T$ of several halo orbits around the $L_{1}$ point of the Earth-Moon system.

\begin{tabular}{|c|c|c|c||c|c|c|c|}
\hline$x(\mathrm{~km})$ & $z(\mathrm{~km})$ & $\dot{y}(\mathrm{~m} / \mathrm{s})$ & $\mathrm{T}($ day $)$ & $x(\mathrm{~km})$ & $z(\mathrm{~km})$ & $\dot{y}(\mathrm{~m} / \mathrm{s})$ & $\mathrm{T}($ day $)$ \\
\hline-316508.9 & 8298.8 & -136.8 & 11.9 & -316541.2 & 13118.0 & -147.0 & 12.0 \\
\hline-316519.0 & 10783.1 & -141.6 & 12.0 & -316577.7 & 15343.8 & -152.6 & 12.0 \\
\hline-316541.2 & 13118.0 & -147.0 & 12.0 & & & & \\
\hline
\end{tabular}
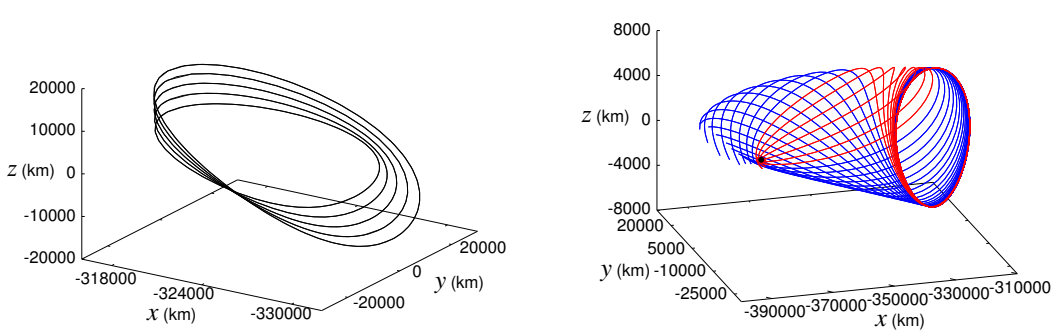

Fig. 5: Left: Halo orbits of class I around the $L_{1}$ point of the Earth-Moon system, associated to the initial conditions given in Table 1. Right: Orbits of the unstable manifold, of the halo orbit with $z$-amplitude equal to $5620.5 \mathrm{~km}$, propagated until their first perilune. The orbits in red collide with the surface of the Moon

for a given displacement $\epsilon$ (typically $\epsilon=10^{-6}$ ), gives the initial condition of two orbits of the unstable manifold, one in each branch of the manifold. We use the one that approaches the Moon. For the globalisation of the manifold, we take the images of the initial condition $\boldsymbol{x}_{h}$ under the flow $\phi_{t}$ associated to the differential equations, $\phi_{t}\left(\boldsymbol{x}_{h}\right)=x_{h}(t)$, together with those of $\boldsymbol{w}^{u}$ under the differential of the flow, $D \phi_{t}\left(\boldsymbol{w}^{u}\right)=\boldsymbol{w}^{u}(t)$. In this way we can obtain a set of orbits of the unstable manifold, equally space in time along the halo orbit, whose initial conditions are,

$$
\left.\boldsymbol{x}_{t}^{u}=\boldsymbol{x}_{h}(t)+\epsilon \boldsymbol{w}^{u}(t), \quad t=t_{0}+(j-1)(T / n)\right), \quad j=1, \ldots, n,
$$

where $T$ is the period of the halo orbit. Some of the orbits of the unstable manifold associated to the first halo orbit in Table 1 are shown in Figure 5.

\subsection{Selection of the inclination $i_{1}$}

It is clear that if we want to target to a polar orbit, the inclination $i_{2}$ achieved by the insertion manoeuvre must be always $90^{\circ}$, but the selection of the suitable inclination $i_{1}$ for the first manoeuvre has more freedom. In this section we study how the value of $i_{1}$ affects the whole transfer cost.

To fix ideas, in a first exploration we keep fixed the initial departing halo orbit (the first one in Table 1), an orbit of its unstable manifold, and the point on the orbit where we do the departure manoeuvre $\Delta \boldsymbol{v}_{1}$. This one corresponds to the first perilune on the orbit. At this point we vary the of value of $i_{1}$ within $\left[i_{x}, 180^{\circ}-i_{x}\right]$, where $i_{x}$ is the angle between $\boldsymbol{r}_{m}$ and the $\left(x^{\prime}, y^{\prime}\right)$ plane. For each value of $i_{1}$ we compute the transfer costs $\Delta v_{1}$ and $\Delta v_{2}$ and $\Delta v_{\text {tot }}=\Delta v_{1}+\Delta v_{2}$. 


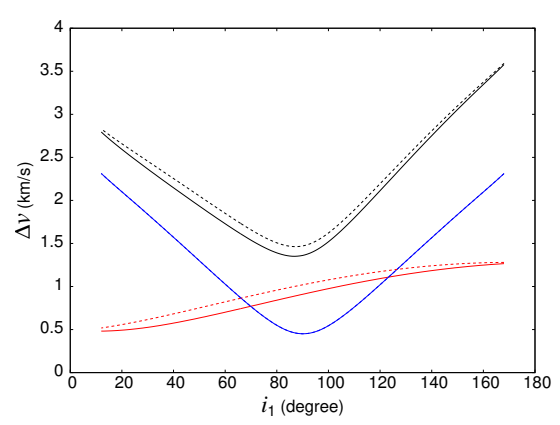

Fig. 6: General behaviour of the transfer costs $\Delta v_{1}$ (red), $\Delta v_{2}$ (blue) and $\Delta v_{\text {tot }}=$ $\Delta v_{1}+\Delta v_{2}$ (black) as a function of the inclination $i_{1}$. The point $P_{1}$ is at a distance from the Moon of $1,0202.31 \mathrm{~km}$, the radius of the polar orbit is of $2037.1 \mathrm{~km}$, and $i_{x}=11.12^{\circ}$, so $i_{1} \in\left[11.12^{\circ}, 168.88^{\circ}\right]$.

Figure 6 shows the behaviour of $\Delta v_{1}, \Delta v_{2}$ and $\Delta v_{t o t}$ as a function of the inclination $i_{1}$ when the target orbit is a lunar polar orbit $\left(i_{2}=90^{\circ}\right)$. The patterns shown in this Figure are almost identical to the ones obtained when other orbits of the unstable manifold are used. There are several things to be mentioned:

1. The value of $\Delta v_{1}$ increases with $i_{1}$, and has an almost constant small slope. Because of this fact, it is better (in terms of $\Delta v_{t o t}$ ) to perform the change of inclination with the departure manoeuvre.

2. The value of $\Delta v_{2}$ has a minimum at $i_{1} \approx 90^{\circ}$ and the curve $\left(i_{1}, \Delta v_{2}\right)$ is almost symmetric with respect to this minimum. This is coherent with the fact that if $\boldsymbol{v}_{1}$ has already done 'almost' all the total change of inclination, the main role of $\boldsymbol{\Delta} v_{2}$ is to do the insertion into the polar orbit.

3. In this example, in which the distance $r_{m}$ from $P_{1}$ to the Moon is not too large, near $i_{1}=90^{\circ}$ the value of $\Delta v_{2}$ is smaller than the one of $\Delta v_{1}$. As we will see later, this is not true if $r_{m}$ is above a certain value.

4. When the inclination of the final orbit is $i_{2} \neq 90^{\circ}$, the symmetry and the minimum properties mentioned in the above item hold for $i_{1}$ equal to $i_{2}$.

5. The two costs associated with the departure manoeuvre $\Delta v_{1}$ (red curves in Figure 6 are very similar, and correspond to the two possible solutions $\boldsymbol{v}_{i-t}^{1,2}$ mentioned in Section 3.2 . The one with larger values of $\Delta v_{1}$ corresponds to the larger values of the angle $\phi_{1}$.

6. For each value of $\Delta v_{1}$ there are two possible values of $\Delta v_{2}$. As was explained in Section 3.4 one of them is always discarded so, instead of having four different $\left(i_{1}, \Delta v_{2}\right)$ curves we have only two. In Figure 6 both curves almost coincide.

7. The near coincidence of the two $\left(i_{1}, \Delta v_{2}\right)$ curves is because $\Delta \boldsymbol{v}_{1}$ is performed at the perilune and the two transfer orbits are almost "symmetric". This is shown in Figure 7 where the latitude of the insertion point $P_{2}$ is displayed in front of $i_{1}$ for the two possible velocities. If the first manoeuvre is done at another point the symmetry is broken and the two costs are different.

8. As it can be seen in Figure 7, the longitude and latitude of the point $P_{2}$ are almost constant (variations less than $0.1^{\circ}$ ), so all the transfer ellipses are almost identical except for a rotation along the $P_{1}-P_{2}$ line. Recall that by construction, the semi-major axis is approximately the segment $P_{1}-P_{2}$ (see Section 3 . 

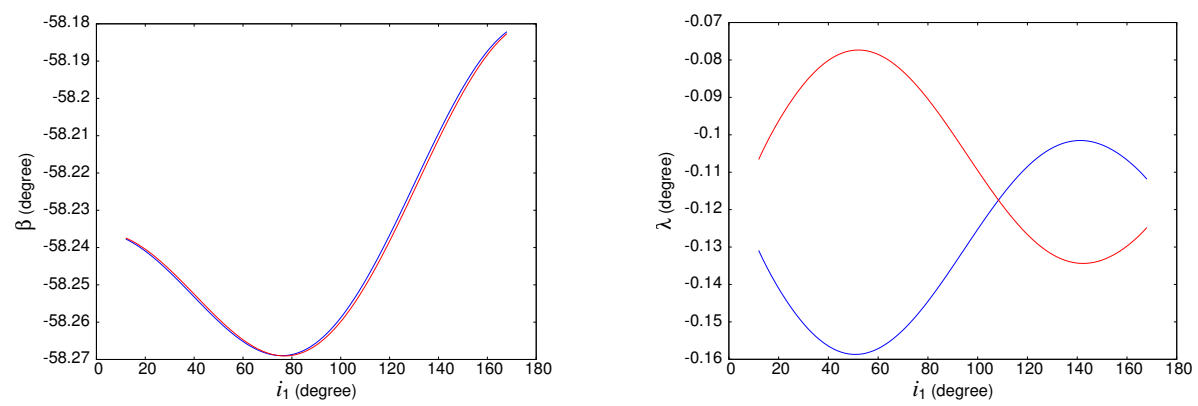

Fig. 7: Location of the longitude $\beta$ (left) and latitude $\lambda$ (right) of the point $P_{2}$ on the sphere $S$, as a function of $i_{1}$ and for the two possible velocities $\boldsymbol{v}_{i-t}^{1,2}$.

Since the initial velocity $\boldsymbol{v}_{i-t}^{\text {sid }}$ of the transfer ellipse is computed in the 2BP model and the integration of the transfer leg is done in the CR3BP framework, the osculating inclination of the transfer ellipse after $\Delta t_{2}$ time units is slightly different (less than $0.2^{\circ}$ ) from the inclination at the departing point of the ellipse. This means that, even if we set $i_{1}=90^{\circ}, \boldsymbol{v}_{f-t}^{\text {sid }}$ is not going to be on the polar plane. The second manoeuvre also takes care of this variation. Note that when $\Delta t_{2}$ is large, the difference of inclinations is also large. This happens when we perform the departure manoeuvre far from the Moon. Figure 8 shows the variation of the inclination along the transfer ellipse with respect to $i_{1}$, together with the change of inclination that must be performed by the second manoeuvre $\Delta \boldsymbol{v}_{2}$.
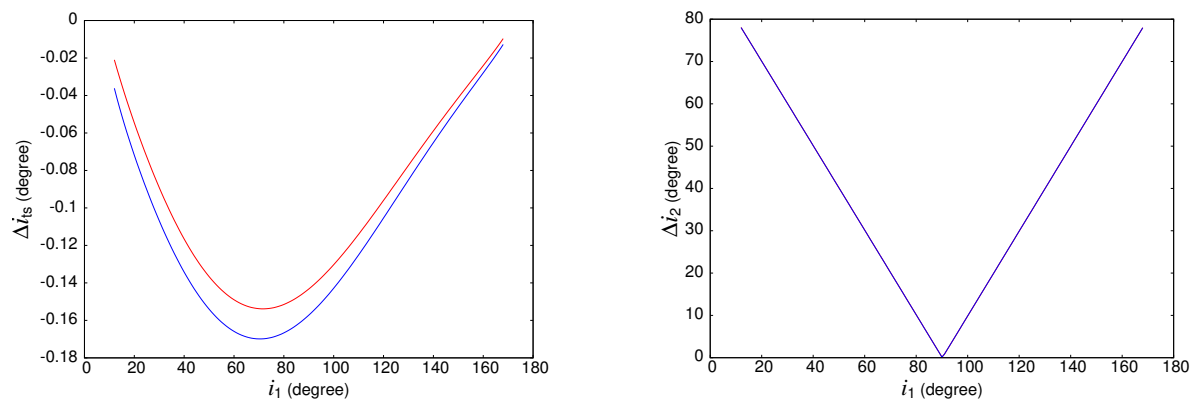

Fig. 8: Variation of the inclination along the transfer ellipse with respect to $i_{1}$ (left), and change of inclination that must be done by the insertion manoeuvre $\Delta \boldsymbol{v}_{2}$ (right). The two curves of the left plot correspond to the two velocities $\boldsymbol{v}_{i-t}^{1,2}$.

4.3 Role of the angles between the arrival and departure velocities at $P_{1}$ and $P_{2}$

At the departure point $P_{1}$, the angles $\phi_{1}^{1,2}$ between $\boldsymbol{v}_{m}$ and the two departure velocities $\boldsymbol{v}_{i-t}^{1,2}$ are closely related to the value of $\Delta v_{1}$. Once $P_{1}$ is selected, the inclination $i_{m}$ of the osculating ellipse is fixed; furthermore, if $P_{1}$ is at the apocentre 
then $\phi_{1}$ is (approximately) equal to $\Delta i_{1}$, in this case $\phi_{1}^{1,2} \approx \Delta i_{1}=i_{1}-i_{m}$, with $i_{1} \in\left[i_{x}, 180^{\circ}-i_{x}\right]$, and, since $i_{m}$ is fixed, the relation between $i_{1}$ and $\phi_{1}^{1,2}$ is linear, as Figure 9 shows. Combining this linear relation with the one between $i_{1}$ and $\Delta v_{1}$ (Figure 6 ) we get the dependence between $\Delta v_{1}$ and $\phi_{1}^{1,2}$, that is displayed in Figure 9. Here the two curves overlap, which demonstrates the dependence of $\Delta v_{1}$ on the angle $\phi_{1}$ when the departure point $P_{1}$ is close to the Moon.
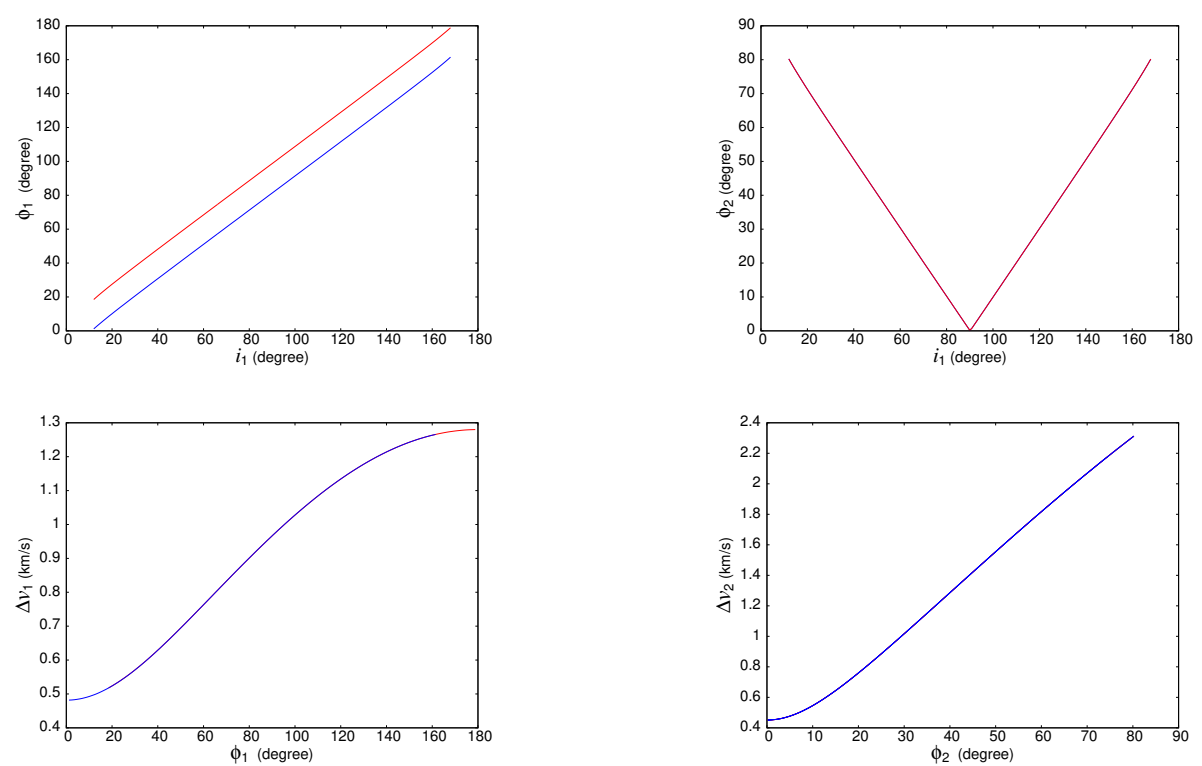

Fig. 9: The top figures display the relation between $i_{1}$ and $\phi_{1}^{1,2}$ (left) and $\phi_{2}^{1,2}$ (right). The bottom figures show the relation of the $\Delta v_{1}$ and $\phi_{1}$ (left), and $\Delta v_{2}$ and $\phi_{2}$ (right)

The constant difference between the two values of $\phi_{1}$ shown in Figure 9 is due to the fact that $P_{1}$ is chosen to be at the first perilune, so the incoming velocity $\boldsymbol{v}_{m}$ of $P_{1}$ is perpendicular to the position vector $\boldsymbol{r}_{m}$; according to (4), the two possible outgoing velocities $\boldsymbol{v}_{i-t}^{1,2}$ are also perpendicular to $\boldsymbol{r}_{m}$ and, as a consequence, the three velocities $\boldsymbol{v}_{m}$ and $\boldsymbol{v}_{i-t}^{1,2}$ are coplanar, so $\left|\phi_{1}^{1}-\phi_{1}^{2}\right|=2 i_{m} \approx 20^{\circ}$. This fact only happens if $P_{1}$ is at the peri/apolune and, at the same time, $P_{1}$ is assumed to be at the apo/pericentre of the transfer ellipse.

The angle $\phi_{2}$ is defined in a similar way as $\phi_{1}$ but at the point $P_{2}$. At this point the modulus of $\boldsymbol{v}_{c}^{\text {sid }}$ is given by $(9)$ and the procedure makes sure that the position vector at $P_{2}$ is perpendicular to $\boldsymbol{v}_{c}^{\text {sid }}$. At $P_{2}$ the velocity along the circular polar orbit is also perpendicular to the position vector $\boldsymbol{r}_{f-t}$, so the angle between the arrival plane and the target plane is exactly the angle between the two velocities. If the departure manoeuvre accounts for the final change of inclination, then the angle $\phi_{2}$ between both velocities is small.

As we can see in Figure 9, the minimum insertion manoeuvre $\Delta v_{2}$ takes place for $i_{1}=90^{\circ}$, since we are aiming at a lunar polar orbit $\left(i_{2}=90^{\circ}\right)$. The trajectory 

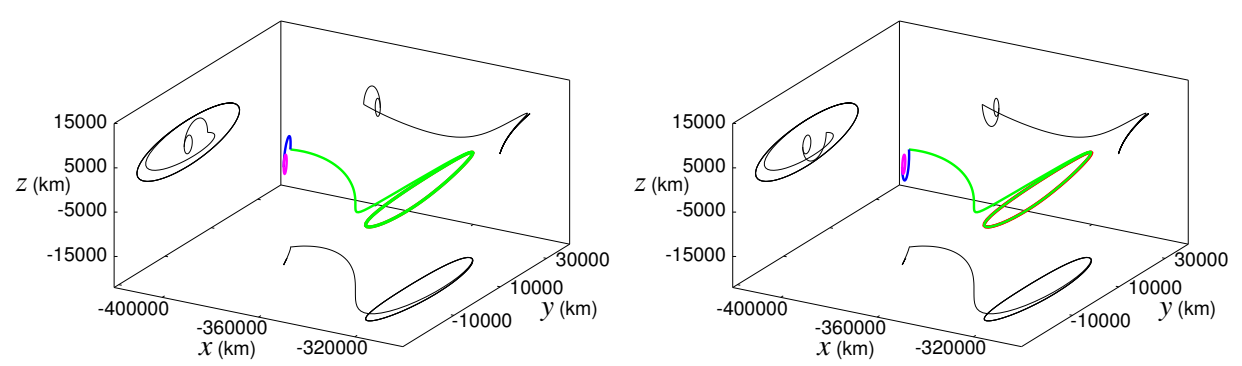

Fig. 10: Transfer trajectories obtained using the two values of $\boldsymbol{v}_{i-t}^{1,2}$, when $P_{1}$ is taken to be the first perilune and $i_{1}=i_{2}=90^{\circ}$.

profiles with minimum transfer costs $\left(i_{1}=90^{\circ}\right)$, obtained with the two possible values of $\boldsymbol{v}_{i-t}$, are displayed in Figure 10. The two Hohmann legs of these transfers are symmetric with respect to the $\left(x^{\prime}, y^{\prime}\right)$ plane because the two $\boldsymbol{v}_{i-t}$ are aligned but pointing to opposite directions. The left plot corresponds to the case that needs less $\Delta v_{t o t}$, which is associated with the smaller $\phi_{1}$. It also corresponds to $\boldsymbol{v}_{i-t}$ with positive $z^{\prime}-$ component, leading to a transfer leg above the $\left(x^{\prime}-y^{\prime}\right)$ plane.

The above analysis shows the strong dependence of the transfer costs $\Delta v_{1}$ and $\Delta v_{2}$ on $\phi_{1}$ and $\phi_{2}$, respectively, when $P_{1}$ is close to the Moon. It suggests that in order to reduce the total transfer cost, the appropriate strategy is to select the desired inclination $i_{1}$ to make $\phi_{2}$ as small as possible. In other words, we should accomplish the main change of inclination manoeuvre at the departure point $P_{1}$. To this end we could set $i_{1}$ to be the final desired inclination, taking polar orbit as the target orbit, we have $i_{1}=i_{2}=90^{\circ}$. This conclusion is the one to be expected considering the a two-body approximation to the problem, in which is better to change the inclination when further from the primary.

From now on, we will only consider the transfer that requires a smaller $\Delta v_{t o t}$.

4.4 The role of the orbit on the unstable manifold with $\Delta v$

In this section we show how the value of $\Delta v$ varies when we consider different orbits of the unstable manifold as 'manifold legs'.

For the halo orbits under consideration, using (13), we have computed several orbits of their unstable manifolds, that have been parametrised by an integer $j=1, \ldots, 25$. The first perilune of each orbit is selected as the departure manoeuvre point $P_{1}$, and we have fixed $i_{1}=i_{2}=90^{\circ}$. Figure 11 shows the distance from $P_{1}$ to the Moon as a function of the index $j$. From this figure it follows that some orbits of the unstable manifold ( $j=1,2,21,22,23,24,25)$ collide the surface Moon before they reach the first perilune. For these orbits we only perform the insertion manoeuvre $\Delta \boldsymbol{v}_{2}$ when the spacecraft reaches the surface $S$. We call them direct insertion transfers, to be distinguished from the usual Hohmann-type transfers.

Figure 12 shows $\Delta v_{1}, \Delta v_{2}$ and $\Delta v_{\text {tot }}$ as a function of the parameter $j$, and as a function of the distance $r_{m}$ from $P_{1}$ to the Moon. The three black segments of the 


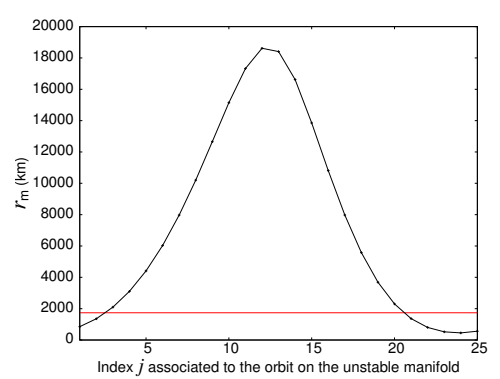

Fig. 11: Distance from $P_{1}$ to the Moon as a function of the index $j$ associated to the family of unstable orbits. The horizontal line indicates the value of the radius of the Moon, $r_{\text {Moon }}=1737.1 \mathrm{~km}$

left plot give the value of $\Delta v_{t o t}$ for these transfers. From this figure it follows that the direct insertion transfers are not a good option in terms of velocity costs, which are all greater than $2.5 \mathrm{~km} / \mathrm{s}$, while for the Hohmann-type ones the cost is much cheaper, less than $2.4 \mathrm{~km} / \mathrm{s}$. A similar fact, about higher costs associated to direct insertion transfers, was also detected and commented in [2]). The minimum cost is of $1.149 \mathrm{~km} / \mathrm{s}\left(\Delta v_{1}=0.619 \mathrm{~km} / \mathrm{s}, \Delta v_{2}=0.530 \mathrm{~km} / \mathrm{s}\right)$ and corresponds to a value of $r_{m}=18407.55 \mathrm{~km}$, which is the largest first perilune distance among all the non-collision orbits of the unstable manifold. This fact will be taken into account later. From now on we focus on the Hohmann-type transfers.
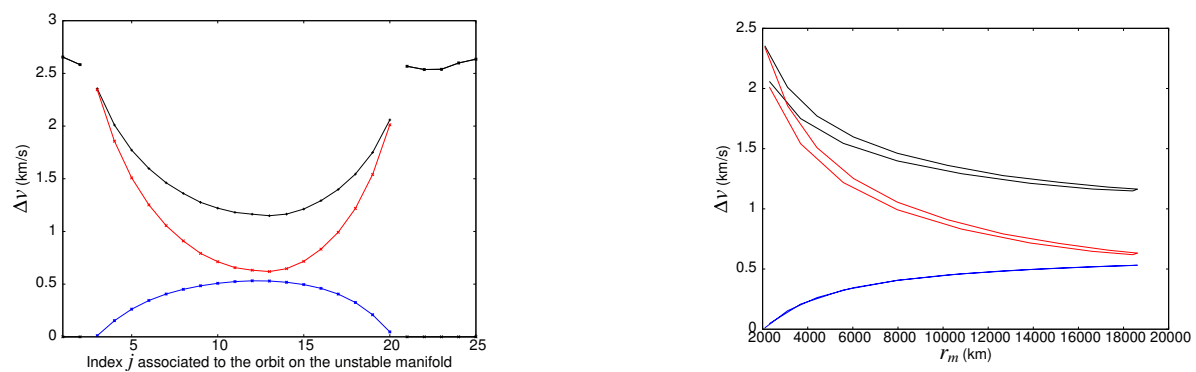

Fig. 12: Transfer costs as a function of the parameter $j$, associated to the family of orbits of the unstable manifold (left), and as a function of the distance from $P_{1}$ to the Moon (right). In both plots $\Delta v_{1}$ is in green, $\Delta v_{2}$ in blue and $\Delta v_{\text {tot }}$ in red.

As it was already noted, the distance $r_{m}$ plays an important role in the transfer cost. With larger $r_{m}$ we get smaller $\Delta v_{1}$ and $\Delta v_{t o t}$, while the insertion manoeuvre $\Delta v_{2}$ shares exactly the opposite change trend. The departure manoeuvre $\Delta v_{1}$ is the dominant part of the total transfer cost when $P_{1}$ is not too far away from the Moon $\left(r_{m} \leq 20,000 \mathrm{~km}\right)$. 
4.5 Varying $P_{1}$ along the manifold leg

Next we want to study the possibility of performing the departure manoeuvre at a point different from the first perilune of the manifold leg. With this purpose we have first analysed the orbits of the unstable manifold that do not collide with the Moon $(j \in[3,20])$. A first exploration allows to classify them in two kinds: those that remain captured by the Moon for at least one revolution $(j \in[15,20])$ and those that escape before performing one revolution $(j \in[3,14])$, which means that there exists no apolune, or that the first apolune is beyond a certain distance.

We have studied the transfers using both kinds of orbits and varying the point $P_{1}$. The departing point $P_{1}$ has been determined integrating forward and backward in time, with time steps of 1 hour, taking as initial condition the perilune point. To be able to use the results of the $2 \mathrm{BP}$ approximation as initial guess, we have set an upper bound of the distance to the Moon $r_{m}$ to be $55,000 \mathrm{~km}$. Figure 13 (left) shows the behaviour of $r_{m}$ for the capture and escape orbits. The minima of this figure correspond to the perilunes and the maxima to the apolunes. In the left plot one can see that the orbit of the unstable manifold performs four revolutions around the Moon, while in the right plot after the first perilune the orbit 'escapes' from the Moon's neighbourhood.
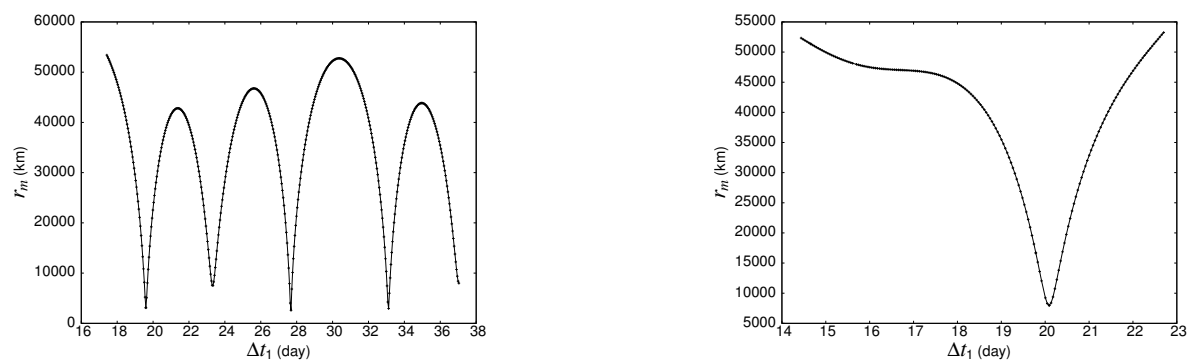

Fig. 13: Behaviour of $r_{m}$ for the capture (left) and escape (right) orbits as a function of the time $\Delta t_{1}$ spent since the departure from the halo orbit.

For the simulations we have fixed $i_{1}=i_{2}=90^{\circ}$ and we show only the results corresponding to one manifold leg of each class. The qualitative behaviour is the same for all the legs of the same class and the quantitative differences are small.

For the two cases under consideration, Figure 14 shows the costs $\Delta v_{1}, \Delta v_{2}$ and $\Delta v_{t o t}$ in front of the time $\Delta t_{1}$ spent since the departure from the halo orbit. In both cases the $\Delta v_{1}$ cost is strongly related to the $r_{m}$ distance, in the sense that the minimum values of the cost are associated to the maxima (apolunes) of the distance to the Moon, as it is clearly shown also in Figure 14. For the capture case it can also be noted that for small values of $\Delta t_{1}$, the first minima of $\Delta v_{t o t}$ (which, in fact, is a global minima) corresponds to the minima of $\Delta v_{1}$, and so, to the apolunes. We will make use of this fact in what follows. The exceptions to this rule (for instance $\Delta t_{1} \approx 29$ days) are associated to larger values of $r_{m}$, in which case the Hohmann leg computed in a $2 \mathrm{BP}$ model is not a good enough approximation of the $\mathrm{CR} 3 \mathrm{BP}$ orbit used for the transfer. In this case, $\Delta v_{2}$ is large 

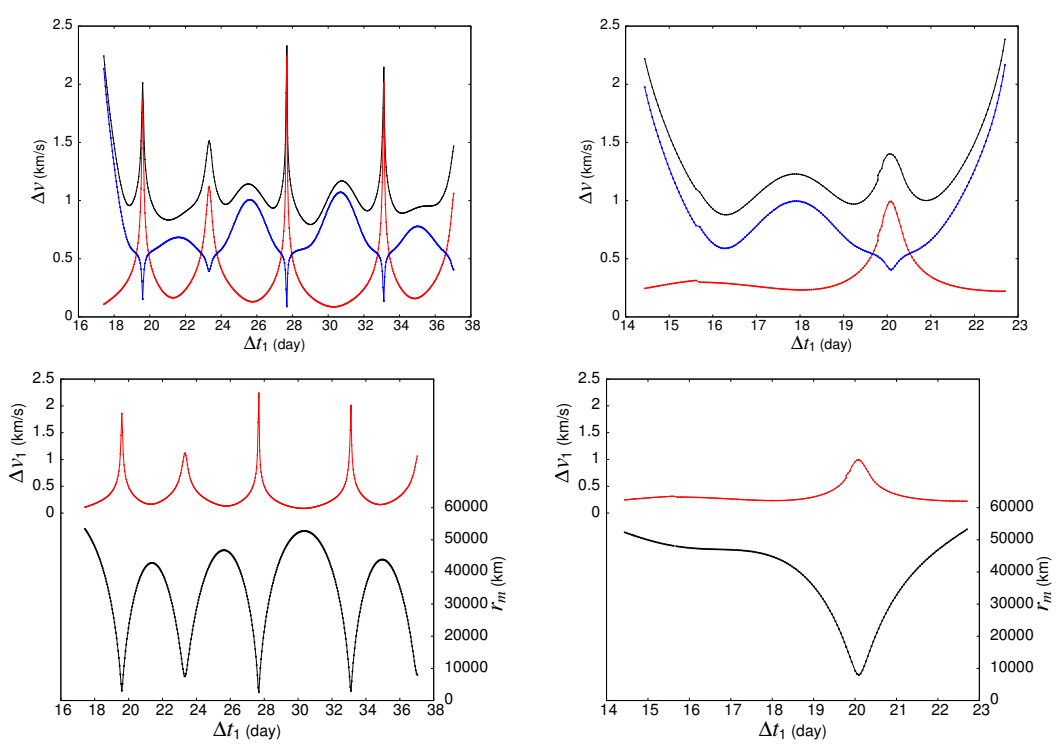

Fig. 14: For the capture case (top left) and the escape case (top right), behaviour of $\Delta v_{1}$ (red), $\Delta v_{2}$ (blue) and $\Delta v_{t o t}$ (black) in front of $\Delta t_{1}$. For both cases, the two bottom figures show the behaviour of $\Delta v_{1}$ (red) and the distance from $P_{1}$ to the Moon (black) in front of $\Delta t_{1}$

since it must also account for a correction of the inclination specified by $i_{1}$ that, due to the approximation, is not achieved by $\Delta v_{1}$.

Looking at Figure 15 we can say that when $r_{m}<20,000 \mathrm{~km}$, the first manoeuvre $\Delta v_{1}$ is the main part of the total $\Delta v$, while for $r_{m}>20,000 \mathrm{~km}, \Delta v_{2}$ becomes larger than the first manoeuvre. This is because when the departure point $P_{1}$ is far from the Moon, a small change in velocity $\boldsymbol{v}_{i-t}$ leads to a big change in the transfer trajectory. Note also that, in this Figure, when we integrate backwards, this is $\Delta t_{1}<19.5$ day, and as $r_{m}$ gets close to the maximum value $55,000 \mathrm{~km}$, $\Delta v_{2}$ increases dramatically while $\Delta v_{1}$ decreases smoothly, so we do not consider to take $P_{1}$ before the first perilune. Moreover, after the intersection between the $\Delta v_{1}$ and $\Delta v_{2}$ curves, the variations of both velocities with $r_{m}$ are smooth up to a certain value $(\approx 45,000 \mathrm{~km})$ and afterwards they become much sharper. As a consequence, the suitable location of the departure point $P_{1}$ should be after the first perilune and with a distance less than 45,000 $\mathrm{km}$ away from the Moon; the first apolune seems to be a good location.

4.6 Setting $P_{1}$ at the first apolune

In this section we explore the situation in which, according to the results of the preceding section, the departure point $P_{1}$ is taken at the first apolune of the manifold leg. In this way we avoid to do unnecessary revolutions around the Moon that, in principle, do not guarantee a decrease of the total $\Delta v_{t o t}$.

Keeping fixed both the departing halo orbit and the target lunar polar orbit, we consider all the captured orbits of the unstable manifold that do not collide with the Moon and that perform at least one revolution around it $(j \in[3,13])$. For all this range of orbits, Figure 16 shows the results that have been obtained. 

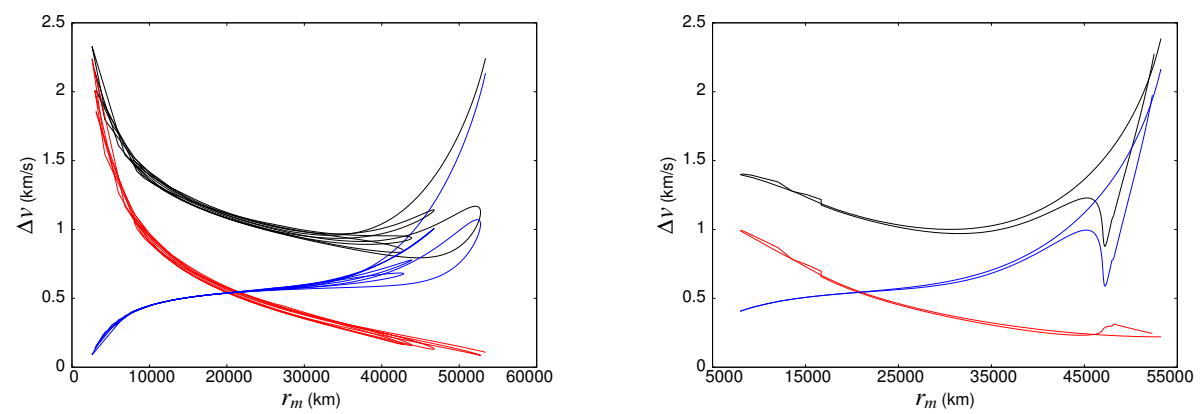

Fig. 15: For the capture case (left) and the escape case (right), behaviour of the costs $\Delta v_{1}$ (green), $\Delta v_{2}$ (blue) and $\Delta v_{t o t}$ (red) in front of the distance $r_{m}$.
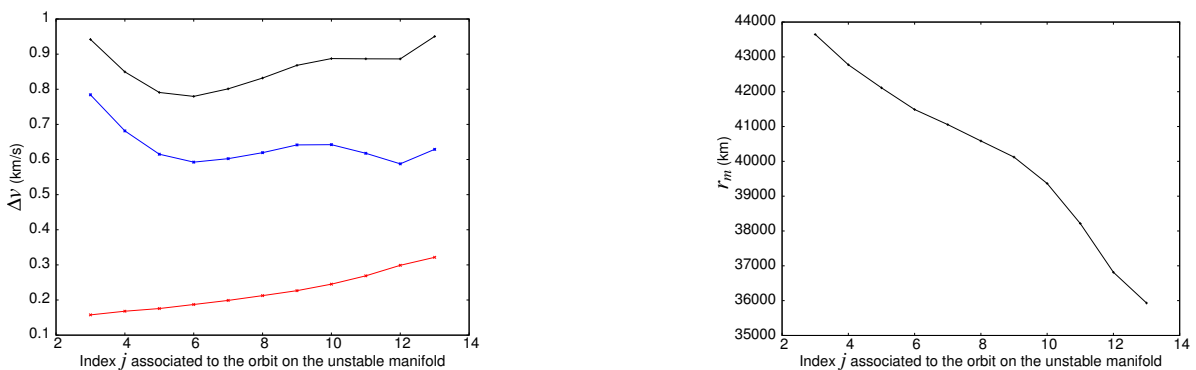

Fig. 16: Behaviour of the transfer costs (left) and distance $r_{m}$ (right) for the captured orbits of the unstable manifold of a halo orbit

As it was already observed, when $r_{m}$ is greater than $37,000 \mathrm{~km}, \Delta v_{2}$ is the dominant part of $\Delta v_{t o t}$. According to Figure 15 , when $r_{m}$ is close to the maximum value $55,000 \mathrm{~km}, \Delta v_{1}$ increases dramatically while $\Delta v_{1}$ decreases smoothly. It has also been observed that the value of $\Delta v_{2}$ is also strongly influenced by the angle $\phi_{2}$, in the sense that the minima of $\Delta v_{2}$ correspond to the minima of $\phi_{2}$. In terms of the total transfer cost, the apolune case requires less than $0.9 \mathrm{~km} / \mathrm{s}$ with a minimum of $0.77977 \mathrm{~km} / \mathrm{s}$, while in the perilune case the minimum is of $1.14915 \mathrm{~km} / \mathrm{s}$.

\subsection{Changing the sizes of the departing and the target orbits}

In this section we study how the size of the initial halo orbit and the radius of the lunar polar orbit affect the transfer cost. For both explorations we keep $i_{1}=i_{2}=90^{\circ}$ as in the preceding sections.

For each halo in Table 1 , we have computed 25 orbits of their unstable manifold, and along each orbit we have performed the departure manoeuvre at both the first apolune and the first perilune.

Figure 17 shows the costs of the transfer as a function of the index $j$ of the orbits of the manifold in the two cases considered, and Table 2 gives the minimum 

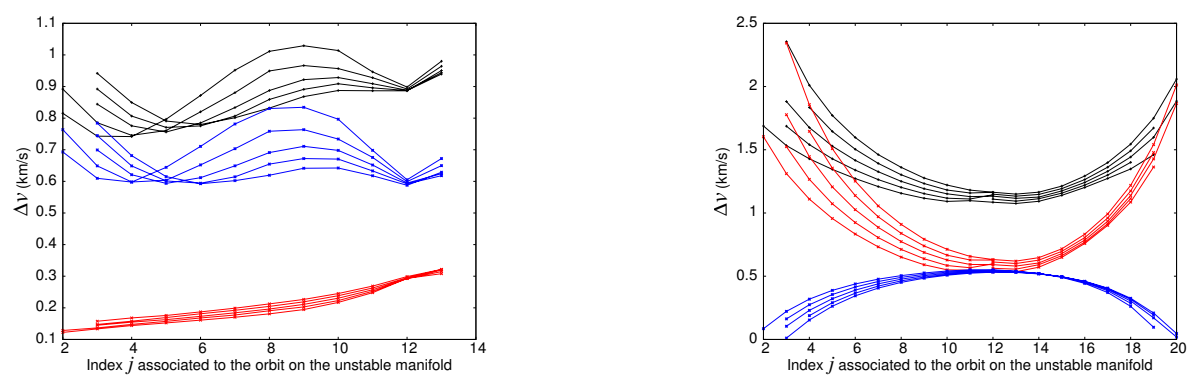

Fig. 17: Transfer costs in the apolune case (left) and perilune case (right) computed using the orbits on the unstable manifolds of the halo orbits given in Table 1. In the left plot the $z$-amplitude increases from top to bottom along the families of curves while in the right plot the sense is reversed.

values of $\Delta v_{t o t}$. Clearly, the pattern behaviours for the $\Delta v^{\prime} s$ are similar to the ones we have already shown for a fixed halo amplitude. In the apolune case, the $z$-amplitude barely plays a role, with merely $30 \mathrm{~m} / \mathrm{s}$ decrease in $\Delta v_{t o t}$, and the minimum transfer cost happens with the smallest $\phi_{2}$. In the perilune case, the minimum transfer cost happens at the point with the biggest $r_{m}$, but a change in the $z$-amplitude of about $10,000 \mathrm{~km}$ only leads to about $70 \mathrm{~m} / \mathrm{s}$ decrease in $\Delta v_{t o t}$ (see Table 2). In the figure, the curves of the apolune case, when seen from top to bottom, correspond to increasing values of the halo-amplitude. In the perilune case, the 'optimal' orbit of the unstable manifold is always the one associated to the upper point of the halo orbit $(j=13)$.

Table 2: Minimum transfer costs in $\mathrm{km} / \mathrm{s}$ for the halo orbits explored.

\begin{tabular}{|c|c|c||c|c|c|}
\hline $\begin{array}{c}z \text {-amplitude } \\
(\mathrm{km})\end{array}$ & $\begin{array}{c}\text { Apolune } \\
\Delta v_{\text {tot }}\end{array}$ & $\begin{array}{c}\text { Perilune } \\
\Delta v_{\text {tot }}\end{array}$ & $\begin{array}{c}z \text {-amplitude } \\
(\mathrm{km})\end{array}$ & $\begin{array}{c}\text { Apolune } \\
\Delta v_{\text {tot }}\end{array}$ & $\begin{array}{c}\text { Perilune } \\
\Delta v_{\text {tot }}\end{array}$ \\
\hline 5620.4505 & 0.77977 & 1.14915 & 13118.0261 & 0.74571 & 1.09389 \\
\hline 8298.7933 & 0.77073 & 1.13316 & 15343.8285 & 0.74168 & 1.07513 \\
\hline 10783.0642 & 0.75623 & 1.11611 & & & \\
\hline
\end{tabular}

The value of the $j$ parameter of the optimal apolune case, which varies between 4 and 6 , indicates that the 'optimal' orbit of the unstable manifold departs close to the right hand corner of the halo, as seen from the Moon.

Table 3: Minimum $\Delta v_{t o t}$ for a fixed departing halo orbit, $j=4$, and target polar orbits of different altitudes

\begin{tabular}{|l|c|c|c|c|c|}
\hline Altitude $(\mathrm{km})$ & 100 & 200 & 300 & 400 & 500 \\
\hline$\Delta v_{\text {tot }}(\mathrm{km} / \mathrm{s})$ & 0.77760 & 0.75894 & 0.74168 & 0.72567 & 0.71076 \\
\hline
\end{tabular}

To complete the analysis, and keeping fixed the departing halo orbit to the one with $z$-amplitude equal to $15343.83 \mathrm{~km}$, we have explored how the changes of the 
size of the lunar polar orbit affect the $\Delta v^{\prime} s$. The results are shown in Figure 18 and Table 3 from which it can be seen that the transfer cost decrease with the altitude of the final polar orbit. Note that in this case: $P_{1}$ is unchanged, $\Delta v_{1}$ is independent of the target polar orbit, since the unstable manifold is unchanged, and $P_{2}$ varies with the altitude of the polar orbit, and so it does the velocity along the manifold leg at $P_{2}$, this explains the results shown in Figure 18 .

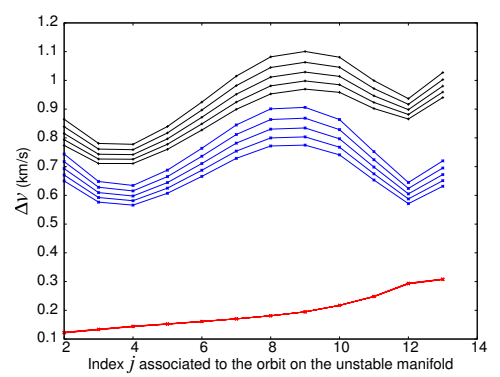

Fig. 18: Transfer costs for a fixed departing halo orbit and 5 lunar polar orbits with different altitudes. The curves of both plots correspond, from top to bottom, to increasing values of $r_{\text {polar }}$.

Finally we have also considered halo orbits of Class II, which are symmetric with respect to the $z=0$ plane of Class I. Of course the results are the same as for Class I. Figure 19 shows the transfer trajectories with the minimum cost obtained for both families where the symmetry with respect to $z=0$ plane between the two families is clearly seen.
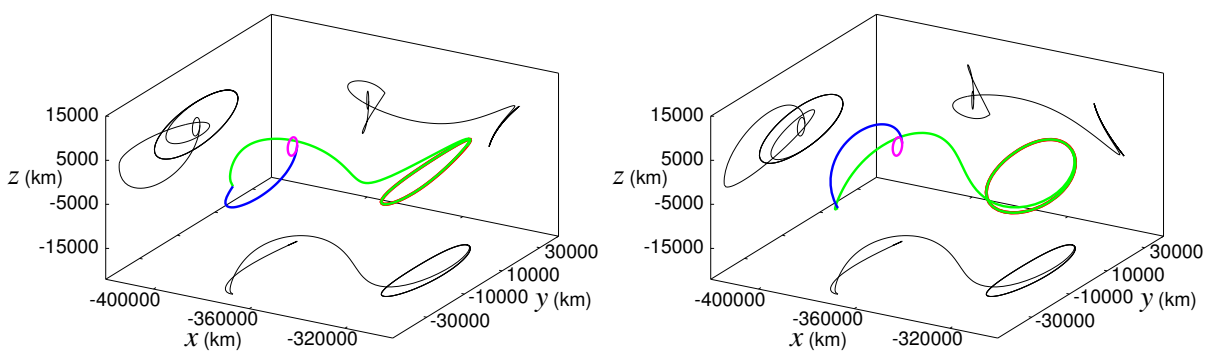

Fig. 19: Transfer trajectory profiles for the minimum cost cases in the apolune case for Class I halo orbit (left) and Class II halo orbit (right).

\section{Conclusions}

In this paper we have done an exploration of the possible parameters that may affect the transfer cost from libration point orbits to lunar orbits. The scenario has 
been the Earth-Moon CR3BP in which we have developed a general procedure to perform transfers from a libration point orbit to a circular polar orbit around the Moon. A key point of the procedure has been the use of the unstable manifold associated to the LPO, which implies that at most only two transfer manoeuvres are required: a departure and an insertion manoeuvre. All the numerical simulations have been done for transfers between halo orbits around the $L_{1}$ libration point and circular lunar polar orbits, although the procedure is general for many other class of orbits.

One of the objectives of the study has been to analyse the role of the different free parameters of the problem in the total transfer cost. The two components of the total transfer cost $\Delta v_{t o t}=\Delta v_{1}+\Delta v_{2}$ have been analysed independently.

Among the parameters, the most important ones are: the distance from the Moon $r_{m}$ at the departure manoeuvre, the inclination $i_{1}$ to be achieved by this manoeuvre, or the change of inclination $\Delta i_{2}$ to be performed by the insertion manoeuvre $\Delta v_{2}$. In general, there is not any parameter playing a dominant role in all the situations considered. However, there are some clear patterns. When departing from a point on the manifold close to the Moon, say $r_{m}<20,000 \mathrm{~km}$, the departure manoeuvre $\Delta v_{1}$ is the dominant cost source and decreases when $r_{m}$ increases. In this situation $\Delta v_{t o t}$ is always greater than $1 \mathrm{~km} / \mathrm{s}$. When $r_{m}>20,000 \mathrm{~km}$, then $\Delta v_{2}$ becomes the dominant part of $\Delta v_{t o t}$, and when $r_{m}$ is greater than $45,000 \mathrm{~km}, \Delta v_{2}$ increases dramatically. The value of $\Delta v_{2}$ is strongly correlated with the change of inclination that the second manoeuvre must perform which, at the same time, is strongly influenced by $r_{m}$ if $i_{1}=i_{2}$. In this situation $\Delta v_{\text {tot }}$ can be smaller than $0.8 \mathrm{~km} / \mathrm{s}$.

It must also be noted that a direct transfer from the departing halo orbit to the lunar polar orbit is not a good option, since it belongs the case in which the departure point along the manifold leg is chosen to be before the first perilune, in this case, the transfer cost $\Delta v_{t o t}$, is always greater than $2.5 \mathrm{~km} / \mathrm{s}$.

As general conclusions we can say that, in order to have low values of $\Delta v_{t o t}$ : the departure point along the unstable manifold must be far from the Moon $\left(r_{m}>\right.$ $20,000 \mathrm{~km})$, but not too far away $\left(r_{m}<45,000 \mathrm{~km}\right)$; in order to make $\Delta v_{2}$ as small as possible, the inclination to be achieved by the first manoeuvre must be set equal to the target final inclination (in the case of a polar orbit, then $i_{1}=90^{\circ}$ ). The size of the departing halo orbit has little influence in the total cost, and if the altitude of the target polar orbit increases then the insertion manoeuvre $\Delta v_{2}$ decreases. Considering that the flight time should not be too long, the departure manoeuvre should be done at the first apolune of the unstable invariant manifold of the LPO.

Acknowledgements Y.C. thanks the support of Doctorate Foundation of Northwestern Polytechnical University and the Chinese Scholarship Council during her stay in Barcelona. G.G. thanks the Catalan grant government for the grant 2014SGR1145, and MINECO-FEDER for the grant MTM2013-41168-P. J.J.M. thanks MINECO-FEDER for the grant MTM201565715-P and the Catalan government for the grant 2014SGR504. The authors would like to thank the referees for a careful reading of the manuscript and their valuable and useful comments. 


\section{References}

1. A Standardised Lunar Coordinate System for the Lunar Reconnaissance Orbiter and Lunar Datasets, LRO Project and LGCWG White Paper, Version 5. NASA 2008. Online available at http://lunar.gsfc.nasa.gov/library/LunCoordWhitePaper-10-08.pdf.

2. E. M. Alessi, G. Gómez, and J. J. Masdemont. Two-Manoeuvres Transfers Between LEOs and Lissajous Orbits in the Earth-Moon System. Advances in Space Research, 45(10):1276$1291,2010$.

3. R. L. Anderson and J. S. Parker. Comparison of low-energy lunar transfer trajectories to invariant manifolds. Celestial Mechanics and Dynamical Astronomy, 115(3):311-331, 2013.

4. B. A. Archinal et al.. Report of the IAU Working Group on Cartographic Coordinates and Rotational Elements: 2009. Celestial Mechanics \& Dynamical Astronomy, 109(2):101-135, 2011.

5. G. H. Born and J. S. Parker. Direct Halo Lunar Transfers. Journal of the Astronautical Sciences, 56(4):441-476, 2008.

6. J. Cao, S. Hu, Y. Huang. Orbit Determination and Analysis for Chang'E-2 Extended Mission. Geomatics and Information Science of Wuhan Universe, 38(9):1029-1033, 2013.

7. P. R. Escobal. Methods of Astrodynamics. J. Wiley, 1969.

8. D. C. Folta, M. Woodard, K. C. Howell, C. Patterson and W. Schlei. Applications of multibody dynamical environments: The ARTEMIS transfer trajectory design. Acta Astronautica, 73:237-249, 2012

9. G. Gómez, J. Llibre, R. Martínez, and C. Simó. Dynamics and Mission Design Near Libration Points. Vol. I Fundamentals: The Case of Collinear Libration Points, World Scientific, 2001.

10. G. Gómez and J.-M. Mondelo. The Dynamics Around the Collinear Equilibrium Points of the RTBP, Physica D: Nonlinear Phenomena, 157(4): 283-321, 2001.

11. G. Gómez, J. J. Masdemont and J.-M. Mondelo. Libration Point Orbits: A Survey From the Dynamical Point of View. In Proc. of the Conference Libration Point Orbits and Applications G. Gómez, M. W. Lo and J. J. Masdemont, Eds. pp. 311-372. World Scientific, 2003.

12. W. S. Koon, M. W. Lo, J. E. Marsden and S. D. Ross. Heteroclinic Connections Between Periodic Orbits and Resonance Transitions in Celestial Mechanics. Chaos, 10:427-469, 2000.

13. W. S. Koon, M. W. Lo, J. E. Marsden and S. D. Ross. Low Energy Transfer to the Moon. Celestial Mechanics and Dynamical Astronomy, 81:63-73, 2001.

14. W. S. Koon, M. W. Lo, J. E. Marsden and S. D. Ross. Dynamical Systems, the Three-Body Problem and Space Mission Design. Marsden Books, ISBN 978-0-615-24095-4, 2011.

15. L. Liu, Y. Liu, J. Cao, S. Hu, G. Tang and J. Xie. CHANGE-2 Lunar Escape Maneuvers to the Sun Earth $L_{2}$ Libration Point Mission. Acta Astronautica, 93:390-399, 2014.

16. J. K. Miller. Lunar Transfer Trajectory Design and the Four Body Problem. 13th AAS/AIAA Space Flight Mechanics Meeting, Paper No. AAS 03-144, 2002.

17. J. S. Parker and R. L. Anderson. Low-Energy Lunar trajectory design. Deep Space Communications and Navigation Series (DESCANSO), 2013.

18. H. Peng, Y. Wang, J.J. Masdemont and G. Gómez. Design and Analysis of Transfers from Lunar Polar Orbits to Sun-Earth Libration Point Orbit. In preparation.

19. Y. Ren and J. Shan. Low-energy lunar transfers using spatial transit orbits. Communications in Nonlinear Science and Numerical Simulation, 19(3):554-569, 2014.

20. Á. Rincón, P. Rojo, E. Lacruz, G. Abellán and S. Díaz. On Non-coplanar Hohmann Transfer Using Angles as Parameters. Astrophysics and Space Science, 359:1-6, 2015.

21. C. E. Roberts. The SOHO mission $L_{1}$ halo orbit recovery from the attitude control anomalies of 1998. In Proc. of the Conference Libration Point Orbits and Applications G. Gómez, M. W. Lo and J. J. Masdemont, Eds. pp. 171-218. World Scientific, 2003.

22. V. Szebehely. Theory of orbits. Academic Press, 1967. 